\title{
Rapid induction of specific associative behavioral memory by stimulation of the nucleus basalis in the rat
}

\author{
Alexandre A. Miasnikov, Jemmy C. Chen, and Norman M. Weinberger ${ }^{*}$ \\ Center for the Neurobiology of Learning and Memory, Department of Neurobiology and Behavior, \\ University of California, Irvine, CA 92697-3800, USA
}

\begin{abstract}
Hypothesized circuitry enabling behavioral memory formation can be tested by its direct activation in the absence of normal experience. Neuromodulation via the cortical release of acetylcholine by the nucleus basalis (NB) is hypothesized to be sufficient to induce specific, associative behavioral memory. Previously, we found that tone paired with stimulation of the nucleus basalis (NBs) for 3000 trials over 15 days induced such memory, supporting the hypothesis. However, as standard associative memory can be established much more rapidly, we asked whether NB-induced memory develops rapidly. Adult male Sprague-Dawley rats, trained and tested in the same calm, waking state, were divided into Paired $(n=5)$ and control $(n=4)$ groups, each of which received a single session of 200 trials of an $8.0 \mathrm{kHz}$ conditioned stimulus (CS) either paired with NBs or with unpaired presentation of NBs. Respiration, cardiac activity, and evoked potentials in the primary auditory cortex $(\mathrm{ACx})$ were recorded. Memory and its degree of specificity were assessed $24 \mathrm{~h}$ later by presenting tones of various frequencies $(1-15 \mathrm{kHz})$ in the absence of NBs to yield behavioral frequency generalization gradients. Behavioral responses to test tones consisted of interruption of ongoing respiration and changes in heart rate. Posttraining behavioral generalization gradients exhibited response peaks centered on the CS frequency for the Paired group alone. Tone evoked potentials from the ACx also developed CSspecific plasticity. The findings indicate that NB induction of specific behavioral associative memory, like normal memory, can develop rapidly and is accompanied by specific cortical plasticity, supporting the view that NB engagement during normal learning produces memory.
\end{abstract}

\section{Keywords}

Learning; Plasticity; Auditory cortex; Association; Evoked potentials; Frequency generalization gradients

\section{Introduction}

Hypothesized circuitry enabling behavioral memory formation can be tested by directly activating its components in the absence of normal experience (i.e., "mimicry," e.g., Martin, Grimwood, \& Morris, 2000). This strong-inference test has been used successfully in delineating cerebellar and related brain-stem pathways subserving the conditioning of eyeblink reflexes (Christian \& Thompson, 2005). However, with one exception (discussed later), such confirming behavioral evidence appears to be absent for neuromodulatory systems, particularly those that directly influence the cerebral cortex.

\footnotetext{
(c) 2005 Elsevier Inc. All rights reserved.

*Corresponding author. Fax: +1 949824 4576., nmweinbe@uci.edu (N.M. Weinberger).
} 
Neuromodulation via the cortical release of acetylcholine (ACh) by the nucleus basalis (NB) has been hypothesized to be sufficient to induce specific, associative behavioral memory (e.g., Weinberger, 1995, 2003; Weinberger et al., 1990). (We use the somewhat redundant phrase "behavioral memory" to distinguish it from neurophysiological plasticity that develops during learning, as the latter is, unfortunately, nowadays often called "memory.") The cholinergic system has long been implicated in learning and memory (Beninger, Wirsching, Jhamandas, \& Boegman, 1989; Deutsch, 1971; Flood, Landry, \& Jarvik, 1981; Hasselmo \& Bower, 1993; Stratton \& Petrinovich, 1963). The nucleus basalis is the major source of cortical acetylcholine (Johnston, McKinney, \& Coyle, 1979; Mesulam, Mufson, Levey, \& Wainer, 1983) and there is extensive evidence of the importance of ACh and of the NB in particular in many aspects of learning and learning-related cortical plasticity (reviewed in Weinberger, 2004b, 2004c). For example, iontophoretic application of ACh to the somatosensory cortex produces long-lasting enhancement of response to sensory stimuli (Dykes, 1990; Metherate, Tremblay, \& Dykes, 1987). Cholinergic agents applied to the primary auditory cortex $(\mathrm{ACx})$ produce long-lasting modification of frequency tuning via muscarinic receptors (Ashe, McKenna, \& Weinberger, 1989; McKenna, Ashe, \& Weinberger, 1989) while pairing a tone with iontophoretic application of muscarinic agonists into the ACx induces pairing-specific, atropine-sensitive, shifts of tuning (Metherate \& Weinberger, 1990). Stimulation of the nucleus basalis (NBs) paired with somatosensory stimulation produces enduring facilitation of responses in the somatosensory cortex (Tremblay, Warren, \& Dykes, 1990; Webster et al., 1991), atropine-sensitive persistent modification of evoked responses in the ACx (Metherate \& Ashe, 1991, 1993), and facilitation of ACx responses to tones (Edeline, Hars, Maho, \& Hennevin, 1994; Edeline, Maho, Hars, \& Hennevin, 1994; Hars, Maho, Edeline, \& Hennevin, 1993). Moreover, cells in the NB develop increased discharges to acoustic conditioned stimuli during tone-shock conditioning before the development of neuronal plasticity in ACx (Maho, Hars, Edeline, \& Hennevin, 1995). Further, both stimulation of the NB and direct application of ACh to the ACx induces CS-specific tuning shifts while lesions of the NB and blockade of muscarinic receptors impair them (Ji, Gao, \& Suga, 2001; Ji \& Suga, 2003; Ma $\&$ Suga, 2003). NB neurons projecting to ACx selectively increase transcription of the gene for ACh's synthetic enzyme, choline acetyltransferase, during tone-shock conditioning, indicating that acoustic learning engages specific cholinergic sub-cellular mechanisms $(\mathrm{Oh}$, Edwards, \& Woolf, 1996). In humans, acoustic conditioning is accompanied by specific plasticity in primary auditory cortex which is blocked by cholinergic antagonists (Morris, Buchel, \& Dolan, 2001; Morris, Friston, \& Dolan, 1998; Thiel, Friston, \& Dolan, 2002).

Classical and instrumental conditioning produce tuning shifts toward or completely to the frequency of the conditioned stimulus (CS), characterized by increased responses to the CS and usually decreased responses to the pre-training best frequency, and increased representation of the CS in the frequency map of primary auditory cortex (Rutkowski \& Weinberger, 2005; Weinberger, 2004b, 2004c). (Complex multitone discrimination protocols are reported to produce generally opposite effects, but do not include behavioral validation of discrimination learning; Ohl \& Scheich, 1996, 1997). The "mimicry" approach has confirmed the hypothesized role of the NB cholinergic system in learning-related specific, associative plasticity in the ACx. Thus, stimulation of the NB cholinergic system can induce receptive field (RF) plasticity that has the same characteristics as behaviorally validated learning-induced auditory cortical plasticity: associativity, CS-specific increased response, rapid induction, discrimination, consolidation (increased strength over time in the absence of more training), and long-term retention (Bakin \& Weinberger, 1996; Bjordahl, Dimyan, \& Weinberger, 1998; Dimyan \& Weinberger, 1999; Miasnikov, McLin, \& Weinberger, 2001). NB-induced RF plasticity depends on the engagement of muscarinic receptors in ACx (Miasnikov et al., 2001). The area representing the frequency of a tone 
paired with NB stimulation is increased in the tonotopic map of the ACx (Kilgard \& Merzenich, 1998a; Kilgard et al., 2001).

While such findings demonstrate that the NB/ACh system can induce ACx plasticity like that which develops during learning, they do not directly address the issue of genuine learning and memory, as behaviorally delineated. Therefore, previously we used the "mimicry" approach by pairing a tone with electrical stimulation of the nucleus basalis for 15 days: 200 trials/day, total of 3000 trials (see also Kilgard \& Merzenich, 1998a, 1998b, 2002; Kilgard et al., 2001). This intervention did induce specific, associative behavioral memory, as indexed by post-training cardiac and respiratory frequency generalization gradients that exhibited maximum responses at the frequency of the CS in the paired group but not the unpaired group (McLin, Miasnikov, \& Weinberger, 2002a, 2003). The findings meet the dual criteria of associativity and specificity that are the long-accepted standards to infer specific associative memory from behavioral change.

However, it is well established that behavioral associative conditioning is normally induced much more rapidly, e.g., within a single training session (Konorski, 1967; Lennartz \& Weinberger, 1992). If NB activation during standard conditioning is sufficient for the formation of specific memory, then NB stimulation should be able to induce memory as rapidly. Therefore, we trained subjects in a single session to determine if NB-induced behavioral memory can do so. We also recorded evoked potentials from the ACx to determine whether the development of NB-induced behavioral memory is accompanied by specific cortical plasticity.

\section{Materials and methods}

\subsection{Subjects and surgery}

The subjects were nine adult male Sprague-Dawley rats (110 \pm 24 days of age, $434 \pm 43 \mathrm{~g}$ weight, mean \pm SD) housed in individual cages with food and water available ad libitum, on a 12/12 h light-dark cycle (lights on at 7:00 a.m.). Following several days of adaptation to the vivarium, animals were handled and learned to sit calmly during later attachment of a thermistor assembly and a cable to their skull pedestal.

Under general anesthesia (sodium pentobarbital, $40 \mathrm{mg} / \mathrm{kg}$ i.p., Abbott Laboratories, North Chicago, IL, supplemented as necessary to maintain areflexia), rats were placed in a stereotaxic frame (David Kopf Instruments, Tujunga, CA) and normal body temperature was maintained. A pedestal, anchored to the skull by stainless steel screws, was built of dental cement (methyl methylacrylate, Co-OralIte Dental Mfg., Diamond Spring, CA) after the scalp was resected and the temporal muscles reflected. Two screws over the frontal sinus served as reference electrodes. Two 10-mm long aluminum hex threaded standoffs were embedded in the pedestal along the rostral-to-caudal axis, bolted to a support and the ear bars removed.

The right primary auditory cortex (ACx) was located by mapping evoked potentials (1-1000 $\mathrm{Hz}, 1000 \times$, DAM-50H, WPI, Sarasota, FL) on the temporal bone (needle point stainless steel electrode) to clicks $(100 \mu \mathrm{s}, \sim 90 \mathrm{~dB}$ SPL, at $0.1 \mathrm{~Hz})$ presented to the contralateral (left) ear via a calibrated miniature speaker. Mapping started at a reference point (AP -4.0, L 8.0; Paxinos \& Watson, 1997; see also Shaw, 1990) and continued until a site was found that had the highest amplitude $(200-400 \mu \mathrm{V})$ response having at least two positive-to-negative components (7-12 ms, P1 and 18-27 ms, N1; 32-60 ms, P2 and 60-104 ms, N2, respectively). The skull was then drilled and an $0.8-\mathrm{mm}$ diameter stainless steel recording epidural screw electrode was inserted and fixed at the identified EP focal point. 
The stimulating electrode was implanted in the ipsilateral (right) nucleus basalis and consisted of a concentric bipolar $13 \mathrm{~mm}$ long stainless steel electrode (type SNEX-100 $\times 13$ $\mathrm{mm}$, David Kopf Instruments, Tujunga, CA). It was lowered through the contralateral hemisphere at a $45^{\circ}$ angle in the frontal plane at AP -2.2 , L 3.2 entering laterally and passing medially. The stimuli were bipolar (pairs of $0.2 \mathrm{~ms}$ long pulses of opposite polarity) electrical stimuli (200-500 $\mu$ A, 200-300 ms long trains, 100 Hz; see Rasmusson, Clow, \& Szerb, 1992), delivered via PSIU6 stimulus isolation units of a Grass S88 stimulator (Grass Instrument, Quincy, MA). The final locus of stimulation for the NB was determined physiologically by obtaining EEG activation from the implanted epidural auditory cortex electrode. Starting $8 \mathrm{~mm}$ below the cortical surface, the stimulating electrode was advanced in $0.2-0.3 \mathrm{~mm}$ steps until, usually at around $10.1 \mathrm{~mm}$ depth along the electrode trajectory, EEG activation lasting several seconds (e.g., 3-8) could be observed consistently in the auditory cortex.

The leads from the thermistor (see Section2.2.1 below), cardiac leads (see Section2.2.2 below), NB stimulating electrode, and epidural cortical recording electrode and their references were crimped to a miniature 9-pin socket imbedded in the plastic pedestal. The signals were conveyed via matching plug of the flexible cable attached to a commutator thus permitting animal's free movements.

All procedures were performed in accordance with the University of California Irvine Animal Research Committee and the NIH Animal Welfare guidelines. During training and testing, subjects were continuously monitored by miniature video cameras.

\subsection{Stimuli, recording, and data analyses}

Training and testing took place while each subject was in a small box $(23 \times 23 \times 31 \mathrm{~cm})$ containing fresh bedding and lined with acoustic-damping tile. This enclosure was contained in a double-walled acoustic chamber (IAC, Bronx, NY). Acoustic stimuli were pure tones (1.0-15.0 kHz, 2 s duration, cosine $10 \mathrm{~ms}$ rise/fall time (10-90\%), $70 \mathrm{~dB}$ SPL) produced by TDT System 3 components (Tucker-Davis Technologies, Alachua, FL) and delivered to a loudspeaker (No. 40-1421, RadioShack, Fort Worth, TX) positioned $35 \mathrm{~cm}$ above the floor of the box. NBs current during training was several times weaker than that used during surgery; it was adjusted with respect to stimulation current threshold as described below.

2.2.1. Respiration-The induction of behavioral memory was assessed by measuring disruption of ongoing respiration pattern and changes in heart rate to various tones following training, as described below. Respiration was detected by a glass-encapsulated thermistor (3 s time constant, $500 \Omega$ at $25^{\circ} \mathrm{C}$, No. 837-5170, Allied Electronics, Fort Worth, TX) attached to a lightweight pedestal-mounted assembly of custom design and fabrication. Care was taken to position the thermistor 1-2 mm lateral of the nares, the location known to have a strongest temperature gradient during exhalation (Wilson \& Sullivan, 1999). The thermistor served as one of the arms of a pre-balanced resistor bridge circuit sensitive to the temperature fluctuations caused by the animal's breathing. The output signal from the bridge was fed to the differential input of a DAM $50 \mathrm{H}$ amplifier $(10,000 \times, 1-100 \mathrm{~Hz}$, WPI, Sarasota, FL). The assembly permitted continual recording of respiration in freely moving rats and caused no discomfort. The amplified signal was digitized by an ADC module (Power-1401 interface, CED, Cambridge, UK) set at 2000 samples/s for roughly 1-Hz/1-s spectral-temporal resolution in off-line calculated FFT functions (see below). The Power-1401 interface was operated under Spike-3 (CED) data acquisition/analysis software; captured data was stored on the hard drive of a Pentium-based PC.

Off-line quantitative processing of respiration started with the calculation of Fast Fourier Transform functions (FFT, Spike-3 software, CED) for a period of $30 \mathrm{~s}, 4 \mathrm{~s}$ immediately 
preceding a trial (Pre), $2 \mathrm{~s}$ during a tone (Dur), and $24 \mathrm{~s}$ after the tone (Post). Major changes in respiration occurred within $0.5-12.5 \mathrm{~s}$ after tone onset. The FFT power was calculated at a frequency resolution of $0.975 \mathrm{~Hz}$ for the power spectra up to $19.045 \mathrm{~Hz}$ for inclusiveness. However, analyses indicated that the respiration signal was almost completely contained within the bandwidth of $0.975-2.925 \mathrm{~Hz}$. The FFT data were used to calculate a "Respiration Power Change Index" (RPCI), on a second-by-second basis. The index was sensitive to both increases and decreases of both frequency and amplitude. RPCIs were calculated as follows: $\mathrm{RPCI}_{i}=\left(\mid \mathrm{Post}_{i}-\mathrm{Pre}\right) /\left(\mathrm{Post}_{i}+\mathrm{Pre}\right)$. A value of zero would indicate no change and a value of 1.0 would indicate complete cessation of respiration. Fig. 1 presents an example of a raw and filtered record, and its RPCI quantification. Respiration, heart rate, and evoked potential data were analyzed with two-way ANOVA (frequency and group factors) and post hoc paired comparisons (Tukey). (Respiration analyses included 13 measurements per trial, across the $13 \mathrm{~s}$ of data captured.) All statistical analyses were accomplished with SPSS v.13 (SPSS, Chicago, IL).

2.2.2. Heart rate-The electrocardiogram (EKG) was obtained from a loop of flexible teflon-insulated stainless steel wire $\left(\sim 001^{\prime} 7\right.$-strand, No. 793200, A-M Systems, Carlsborg, WA). Insulation was removed from a $\sim 5 \mathrm{~mm}$ section and the loop was threaded with a $5 \mathrm{~cm}$ stainless steel guide subcutaneously from behind the neck through the thorax such that the un-insulated section was located in the chest adjacent to the heart. The EKG was recorded with reference to a stainless steel screw in the skull.

The EKG/EMG signal was amplified and filtered (DAM 50H amplifier $(1000 \times$, band-pass 10-100 Hz), digitized at 500 samples/s with Power1401/ Spike-2 software and processed off-line. An amplitude threshold was set to separate the higher amplitude EKG from EMG activity that was detected, based on the amplitude distribution histograms calculated for each animal individually. The instantaneous heart rate (expressed in beats-per-minute, BPM) was derived from consecutive inter-beat intervals (IBIs). The tone-elicited heart rate responses were calculated by subtracting the maximal IBI from minimal IBI on each trial. Fig. 2 presents a sample record and its quantification. Statistical analyses were the same as for respiration except that each trial contributed one measurement (peak-valley) for cardiac responses.

2.2.3. EEG and evoked potentials-The epidural $\mathrm{ACx}$ signal was amplified and filtered (DAM-50H amplifier, $1000 \times$, band-pass $1-1000 \mathrm{~Hz}$ ), digitized at $500 \mathrm{samples} / \mathrm{s}$ with Power1401/Spike-2 software, and processed off-line. The FFT power was calculated off-line at frequency resolution of $0.975 \mathrm{~Hz}$ for the power spectra up to $59.965 \mathrm{~Hz}$. The FFT data were used to calculate an EEG "Power Change Index" (EEG PCI), on a second-bysecond basis for each EEG frequency band separately as follows: delta, 0.98-2.92; theta, 2.93-8.78; alpha, 8.79-14.62; beta1, 14.63-20.47; beta2, 20.48-33.15; gamma, 33.1659.97. The index was sensitive to both increases and decreases in EEG Power. EEG PCIs were calculated as follows: EEG PCI $i=\left(\right.$ Post $\left._{i}-\mathrm{Pre}\right) /\left(\mathrm{Post}_{i}+\right.$ Pre $)$. A negative value would indicate a decline and a positive value would indicate a rise in power relative to its baseline.

Evoked potentials to tone onset were recovered from the EEG records off-line (Fig. 3). They consisted of P1 (maximal amplitude within 5-15 ms time window), N2 (minimal amplitude within 16-34 ms time window), P2 (35-55 ms), N2 (56-85 ms), and often several more components, notably, P3 (86-121 ms), N3 (122-160 ms), and, least stable, P4 (161-250 $\mathrm{ms})$. However, we report here only findings for the P1 and N1 components, because they are generated by elements in the primary sensory cortices (Barth, Kithas, \& Di, 1993; Shaw, 1988) and they exhibit frequency tuning (Galván, Chen, \& Weinberger, 2001). The P1-N1 amplitude (peak to valley) was calculated separately for each tone during presentation of test frequencies pre- and post-training. 
2.2.4. Animal state-To achieve maximum sensitivity to behavioral responses, it was desirable to monitor state. During training and test sessions, on-line respiration, EKG/EMG, and EEG data were used to estimate behavioral and physiological state (Fig. 4). Prior observation of subjects during an initial session of accommodation to the experimental chamber showed that respiration waveforms were highly irregular in frequency and amplitude during exploratory behavior/grooming, accompanied by high amplitude EMG and low amplitude high frequency EEG. During REM sleep, respiration was somewhat more regular while accompanied by low amplitude EMG and low amplitude high frequency EEG. In contrast, the respiration was highly regular during the calm waking state, typically when the animal was sitting quietly with its eyes opened. Therefore, we presented tones and NB stimulation only when respiration tended to be regular, thus providing for a stable pre-trial behavioral baseline against which changes in respiration and heart rate could be measured. Stimulation of the NB (during training) outside both the exploration and REM states was particularly important because cortical ACh levels during these states may be very high (Jasper \& Tessier, 1971; Jimenez-Capdeville \& Dykes, 1993) and the EEG highly activated, thus potentially involving a ceiling effect, i.e., little or no change in cortical state to NB stimulation.

\subsection{Experimental design}

The subjects were assigned to two groups: Paired CS and NB stimulation $(\mathrm{P}, n=5)$ and a group that received unpaired presentation of these stimuli $(\mathrm{Up}, n=4)$. The groups differed neither in age $(p>.07)$ nor weight $(p>.08$, two-tailed $t$ test). After 7-13 days of recovery from surgery, NB stimulation threshold was determined in the un-anesthetized state. After placement in the experimental chamber, each animal typically explored and groomed before going into a state of quiet waking, characterized by a recumbent posture, and an EEG containing high amplitude slow-wave activity, low amplitude EMG, and regular respiration (Fig. 4B). NB stimulation (see above) was delivered every few minutes at increasing levels starting $\sim 30 \mu \mathrm{A}$ ( $100 \mathrm{~Hz}$ bipolar, $200 \mathrm{~ms}$ train) until stimulation reliably elicited a $\sim 3-8 \mathrm{~s}$ epoch of cortical activation (decrease in low frequency activity often accompanied by increase in gamma activity). The current levels used in subsequent training with NB stimulation did not elicit any movements. There was no significant difference between groups in current levels $(\mathrm{P}=67.6 \pm 11.3 \mu \mathrm{A}, \mathrm{Up}=63.6 \pm 5.4 \mu \mathrm{A}, p>.50$ two-tailed $t$ test $)$.

To induce and subsequently evaluate stimulus-specific behavioral memory, we used the approach of acquiring behavioral baseline responses to many frequencies, then training with one frequency and testing the training effects with many frequencies (behavioral frequency generalization gradients). In such training, successful stimulus-specific conditioning would be indexed by non-flat frequency generalization gradients with peaks at or near the frequency of the CS. The main protocol required four consecutive days (Fig. 5): Days 1 and 2, pre-training baseline generalization; Day 3, pairing of the CS with NB stimulation or unpaired presentation of the "CS" and NB stimulation; Day 4, post-training generalization. The first baseline generalization session (Day 1) was used to acclimatize subjects to the testing environment and thus data from this session were not analyzed. Although not essential, contextual transfer between training and generalization testing sessions was reduced by delivering animals to the lab via different circuitous routes and training them in the dark (red light) in one acoustic chamber but testing in the light in another acoustic chamber. To quantify the activation effect of NBs alone, we also conducted an additional experimental session for the Paired group (Day 5), during which the CS and NBs were presented unpaired.

During training, the Paired group received 200 trials of tone $(8.0 \mathrm{kHz}, 2 \mathrm{~s}, 70 \mathrm{~dB}$ SPL) followed by NBs (same level as determined post-operatively) that overlapped CS 
presentation and co-terminated with CS offset (i.e., the CS-US interval was $1.8 \mathrm{~s}$ ). The Unpaired group received 200 tones and $200 \mathrm{NBs}$ in random order with the restrictions that no more than three of the same type could occur consecutively and that NBs could not occur during a $15 \mathrm{~s}$ period either following or preceding presentation of the CS; this prevented both stimuli overlap and "near trace" forward or backward pairing. Inter-trial intervals during training sessions averaged $80 \mathrm{~s}$ and were not significantly different between the groups $(p>.25$, two-tailed $t$ test).

On frequency generalization test days, subjects received unpaired presentation of tones of nine different frequencies $(1.00,2.75,4.50,6.25,8.00,9.75,11.50,13.25$, and $15.00 \mathrm{kHz}$, $70 \mathrm{~dB}$ SPL, no more than two stimuli of the same frequency in a row) for 200 trials total. Inter-trial intervals (ITI) averaged $94 \mathrm{~s}$ and did not differ significantly between the groups $(p$ $>$.85). The minimum ITI was randomly selected by the computer from the range of 30-60 s, to prevent a stimulus from overlapping onto the effects of previous stimulation. Upon the passage of a minimum ITI, the experimenter began looking for stability in respiration waveform (lasting for at least $4 \mathrm{~s}$ ) before an appropriate stimulus was allowed to be presented. Across subjects the range of the same tone given during a session was from 10 to $34(21.8 \pm 4.3)$. The number of same tones presented averaged $22.2 \pm 4.3$ and was not significantly different between groups ( $p>.15$, Levene's test for Equality of Variances). There was no difference between groups in the number of tones of any of the nine frequencies presented (two-way ANOVA: groups, $F_{1,225}=2.272, p=.133$; test frequencies $(1-15 \mathrm{kHz}): F_{8}, 225=.892, p=.523$; interaction: $\left.F_{8}, 225=.976, p=.455\right)$. The groups were not statistically different either in time of the day the experiments began $(p>.30)$, or in the length of experimental sessions, which ranged from 4.75 to $8.0 \mathrm{~h}$ across subjects $(p>.85$, two-tailed $t$ tests).

\subsection{Histology}

Following termination of the experiments, an electrolytic lesion (4 ms pulses at $100 \mathrm{~Hz}, 500$ $\mu \mathrm{A}$ for $60 \mathrm{~s}$ ) was made with bipolar current through the stimulating electrode while the animal was under sodium pentobarbital anesthesia. It was then given an overdose of sodium pentobarbital and perfused through the heart with saline followed with $10 \%$ paraformaldehyde in $0.1 \mathrm{M}$ phosphate buffer ( $\mathrm{pH}$ 7.3). The brain was removed and coordinates of the recording electrode on the skull were measured from Bregma. Following several days of post-fixation in paraformaldehyde solution with $0.8 \mathrm{M}$ sucrose added for subsequent cryoprotection, the brain was sectioned at $50 \mu \mathrm{m}$ with freezing microtome, sections mounted onto gelatin-coated slides, dried, and stained for Nissl substance to determine stimulation sites.

\section{Results}

\subsection{Stimulation and recording sites}

Stimulation loci are summarized in Figs. 6A-C. All electrodes were located within the region of the caudal nucleus basalis, along the ventro-lateral edge of the internal capsule and ventral portion of the lateral globus pallidus, as projected on standardized outlines of sections from the atlas of Paxinos and Watson (1997) at anterior-to-posterior (AP) ranging from 1.3 to $2.3 \mathrm{~mm}$ posterior to Bregma, medio-lateral (L) ranging from 2.4 to $4.2 \mathrm{~mm}$ lateral to Midline, and depth (D) coordinates ranging from 6.7 to $7.7 \mathrm{~mm}$ (AP: $-1.7 \pm 0.5$ $\mathrm{mm}, \mathrm{L}: 3.0 \pm 0.6 \mathrm{~mm}, \mathrm{D}: 7.3 \pm 0.3 \mathrm{~mm}$ ). There was no statistical difference in average loci of stimulation between groups ( $t$-tests: AP: $\mathrm{P}=-1.6 \pm 0.5 \mathrm{~mm}, \mathrm{Up}=-1.8 \pm 0.6 \mathrm{~mm}, p>$. $55 ; \mathrm{L}: \mathrm{P}=2.9 \pm 0.5 \mathrm{~mm}, \mathrm{Up}=3.2 \pm 0.8, p>.45 ; \mathrm{D}: \mathrm{P}=7.3 \pm 0.2 \mathrm{~mm}, \mathrm{Up}=7.3 \pm 0.4 \mathrm{~mm}$, $p>.90)$. 
The coordinates of the epidural recording screws are shown in Fig. 6D. The positions of different cortical cytoarchitectonic areas centered around the ACx were derived from the frontal section outlines reported in (Paxinos \& Watson, 1997). There was no statistical difference in recording loci between groups (AP: $\mathrm{P}=-4.5 \pm 0.5 \mathrm{~mm}, \mathrm{Up}=-4.8 \pm 0.7 \mathrm{~mm}, p$ $>.55 ; \mathrm{L}: \mathrm{P}=8.9 \pm 0.5 \mathrm{~mm}, \mathrm{Up}=8.6 \pm 0.4, p>.40, t$-tests $)$.

\subsection{Effects of training on behavior}

3.2.1. Respiration-The respiration findings are presented in Fig. 7. Baseline responses to various frequencies (i.e., the magnitude of interruption of respiration across groups) differed significantly (two-way ANOVA, $F=56.637, d f=8, p<.001$ ). The largest responses were in the range of $4.5-9.75 \mathrm{kHz}$, as expected from the behavioral audiogram which shows that rats have a high level of sensitivity to this frequency range (Heffner, Heffner, Contos, \& Ott, 1994). More importantly, baseline responses to tones were not significantly different between groups (two-way ANOVA, Group: $F_{1,22,732}=.16, p=.899$ ) (Fig. 7A).

In contrast to the baseline findings, post-training generalization gradients revealed significant differences in response to tones between the Paired and Unpaired groups (twoway ANOVA, Groups: $\left.F_{1,23,382}=82.861, p<.001\right)$. Post hoc Tukey's tests revealed the presence of significant differences between responses at and around the $8.0 \mathrm{kHz}$ CS frequency; responses at and immediately adjacent to the CS frequency were larger in the Paired vs. the Unpaired group (6.25 kHz: $p<.001,8.00 \mathrm{kHz}: p<.001,9.75 \mathrm{kHz}: p<.05)$. Across groups, there remained a significant difference in responses to different tones $\left(F_{8}, 23,382=69.843, p<.001\right)$. There was also significant interaction between the two factors post-training $\left(F_{8}, 23,382=22.882, p<.001\right)$. This appears to reflect an increase of the magnitude of respiratory responses to the CS and immediately adjacent frequencies but decreased response magnitude in the Unpaired group (Fig. 7B).

A within-groups comparison of the baseline and post-training responses for each test frequency supports this conclusion (Fig. 7C). The largest changes in post-training vs. pretraining responses occurred at and around the CS frequency. Statistically significant response enhancement in the Paired group developed at 6.25 and $8.00 \mathrm{kHz}$ (Tukey's test, all $p<.001$ ). (The response to $2.75 \mathrm{kHz}$ also increased, $p<.01$ ). The Unpaired group developed a significant decrease at the training frequency of $8.00 \mathrm{kHz}(p<.001)$. This may reflect specific habituation to the repeated "CS" frequency (Section 4).

3.2.2. Heart rate-The effects of training on heart rate change were comparable to those for respiration (Fig. 8). During the baseline period, there was no significant difference between groups $\left(F_{1,1736}=.024, p=.878\right)$. There was a difference in responses to different tone frequencies across groups $\left(F_{8}, 1736=8.233, p<.001\right)($ Fig. $8 \mathrm{~A})$.

Training modified behavioral generalization gradients in both groups. During the posttraining period, a two-way ANOVA showed that responses in the two groups differed significantly $\left(F_{1,1781}=58.561, p<.001\right)$. Post hoc Tukey's tests revealed the presence of significant differences at and adjacent to the $8.0 \mathrm{kHz} \mathrm{CS}$ frequency; responses were larger in Paired group at $6.25 \mathrm{kHz}(p<.003)$ and $8.00 \mathrm{kHz}(p<.005)$ (Fig. 8B). As expected, across groups, the significant difference in response to various frequencies remained $\left(F_{8,1781}=\right.$ 13.191, $d f=8, p<.001)$. However, there was no significant interaction between the two factors post-training $\left(F_{8}, 1781=1.013, d f=8, p=.424\right)$.

The within-groups comparison of the pre- and post-training responses revealed no significant change in the magnitude of the cardiac response to any single tone frequency (Fig. 8C). However, grouping the nine test frequencies into consecutive groups of three frequencies did reveal significant CS-related effects. (Although grouping reduced the 
spectral resolution of analysis, it increased the test sensitivity by increasing the number of measurements within each comparison). The Paired group exhibited enhancement of responses centered at the CS frequency $(6.25,8.00$, and $9.75 \mathrm{kHz}: p<.001$, Tukey's test). Principal changes in the Unpaired group were a decline in responses at the same frequencies $(6.25,8.00$, and $9.75 \mathrm{kHz}: p<.004)$. Similar to decreased responses in respiration centered on the "CS" frequency, these frequency-specific decreases probably reflect habituation.

\subsection{Auditory cortical evoked potentials}

A two-way ANOVA indicated that baseline responses to the various tone frequencies were significantly different across groups $\left(F_{8}, 1730=5.138, p<.001\right)$ but the amplitude of auditory evoked potentials across frequencies did not differ between groups $\left(F_{1,1730}=\right.$ 2.397, $p=.122$ ) (Fig. 9A). The interaction between frequencies and groups was not significant $\left(F_{8}, 1730=.619, p=.762\right)$. Although responses to various tones differed, no clear tuning (i.e., no systematic relation to frequency) was evident. This most likely reflects the poor spatial resolution of the low-impedance epidural screw electrodes (Fig. 6). As the goals of this experiment did not include the study of associatively induced tuning shifts toward/to the CS frequency (Bakin \& Weinberger, 1990; Edeline, Pham, \& Weinberger, 1993; Edeline \& Weinberger, 1992; Galván \& Weinberger, 2002) the lack of frequency tuning is not inimical to the present findings.

After training, the amplitudes of evoked potentials to different frequencies across groups were still significantly different $\left(F_{8,1782}=8.143, p<.001\right)$. More importantly, evoked potentials were significantly different between groups after training $\left(F_{1}, 1782=12.158, p=\right.$. 001) (Fig. 9B). Although post hoc Tukey's test did not reveal significant group differences for any individual frequencies, there still were detectable changes in the vicinity of the CS. Clustering consecutive frequencies into groups of three showed statistically significant differences centered on the CS frequency $(6.25,8.00$, and $9.75 \mathrm{kHz}: p<.04)$. Whereas it happened that baseline responses to the CS and adjacent frequencies were all smaller for the Paired vs. the Unpaired Group, this relationship was reversed after training (Figs. 9A and B). There was no significant interaction between the frequency and group factors posttraining $\left(F_{8}, 1782=1.251, p=.265\right)$. Within-groups differences (baseline vs. post-training) were not significant for any frequency despite the general increase in response to CS-related frequencies in the Paired group and the decrease in response in the Unpaired group (Fig. 9C).

The relationship between the effects of training on evoked potentials to the CS and immediately adjacent frequencies $(6.25,8.00$, and $9.75 \mathrm{kHz})$ and behavior could be investigated directly as each subject yielded both types of data. Therefore, we performed separate correlational analyses on the magnitude of change of these evoked potentials with respect to the magnitude of training-induced changes in both respiration and heart rate. This analysis was performed across groups because significant (but opposite) effects were evident for both the Paired and Unpaired groups at and near the CS frequency. The analyses involved 27 data points for each correlation ( 3 frequencies $\times 9$ subjects). There was a significant positive relationship between the changes in respiration and the changes in evoked potential amplitude $\left(r_{\mathrm{xy}}=.49, p<.02\right)$ (Fig. 10A) and also between changes for heart rate and evoked potential amplitude $\left(r_{\mathrm{xy}}=.50, p<.01\right)$ (Fig. 10B). The findings indicate that training which induces specific behavioral memory also induces similar changes in the response of the ACx.

\subsection{Control for effectiveness of NB stimulation}

NB stimulation may serve as an effective unconditioned stimulus because it can elicit unconditioned changes in respiration and heart rate (McLin, Miasnikov, \& Weinberger, 
2002b). As the NB appears to be part of an activating system, respiratory and cardiac effects may reflect either or both increased energetic demands and efferent components of the orienting reflex (Sokolov, 1958). As tone paired with NB stimulation induced CS-specific increased behavioral responses in the Paired group, but unpaired presentation of tone and NBs failed to do so, the pairing effects seem to be associative. However, it might be argued that the differential results reflect differentially effective NBs. That is, if NB stimulation were effectively weaker for the Unpaired group, then the success of the Paired group could not be attributed necessarily to associative factors. We have already noted that there was no significant difference between groups in the mean current levels of NBs ( $p>.50$ two-tailed $t$ test) and there was no significant difference in the loci of stimulation ( $p>.45$, Figs. 6A-C) and recording $(p>.40$, Fig. 6D) sites.

Nonetheless, none of these factors provide a direct measure of the physiological effectiveness of NBs. Therefore, we analyzed the effect of NBs on the EEG spectrum for all training trials. Fig. 11 presents examples of raw and bandpass filtered EEG for subjects in the Unpaired (Fig. 11A) and Paired (Fig. 11B) groups. Spectral analyses for responses to NBs for all training trials in the Unpaired group are given in Fig. 11C. Note that NBs produced some increase in gamma with predominant decreases in lower frequency bands (most notably in theta, alpha, and beta1), as previously reported (McLin et al., 2002b; Rasmusson et al., 1992). (The delta band data are not shown because NBs itself produces a very large cholinergic potential whose dominant spectrum is within the low frequency delta band (Metherate, Cox, \& Ashe, 1992).) However, an equivalent analysis could not be performed for the Paired group because NBs was necessarily preceded by presentation of the conditioned stimulus tone $(8.0 \mathrm{kHz})$ on every trial. Moreover, it is known that pairing tone with NBs produces conditioned EEG activation in response to the conditioned stimulus (McLin, Miasnikov, \& Weinberger, 2003). Therefore, the EEG spectrum would be expected to exhibit greater effects during pairing because of the combined activating effects of both the conditioned stimulus and NBs. Indeed, an analysis of the EEG during tone-NB stimulation in the Paired group confirmed this prediction (Fig. 11D).

To determine the unconfounded effects of NBs on the EEG of the Paired group, it was necessary to test them in the absence of preceding tone presentation. Thus, following conclusion of post-training testing (Fig. 5, stage 5), we stimulated this group in a manner identical to the training of the Unpaired group, i.e., a single session of tone and NB stimulation presented unpaired, using the same NB current levels as had been employed during pairing. (This may have constituted an extinction session, analysis of which will be reported elsewhere.) The results are presented in Fig. 11E. Note that NB stimulation alone did produce activation, principally a pronounced decrease in frequency bands lower than gamma (theta, alpha, beta1, and beta2). Note, however, that NBs in this evaluation did not have a larger EEG effect than did the Unpaired group (Fig. 11C). Actually, it was statistically smaller for alpha $(p<.02)$, beta1 $(p<.05)$, and gamma $(p<.03)$. Thus, memory induction (and evoked potential physiological plasticity) in the Paired group were not caused by more effective NBs in the Paired vs. Unpaired group, but rather by associative processes.

\section{Discussion}

\subsection{Rapid NB induction of specific behavioral memory}

The major goal of this study was to determine whether or not specific associative behavioral memory that is induced by pairing a tone with stimulation of the nucleus basalis can develop rapidly. Such memory would be evident by CS-specific changes in frequency generalization gradients, most likely an increase in response as this has been repeatedly found in classical and instrumental conditioning (Weinberger, 2004b). In the present case, "rapidly" refers to a single training session of 200 trials, which is a brief time compared to the prior study that 
employed 200 trials per day for 15 days (McLin et al., 2002a). They found post-training CSspecific frequency generalization for respiration and heart rate, i.e., increased responses at the peak of the generalization gradients. The absence of such specific functions in a group receiving unpaired tone and NB stimulation showed that the memory induction was associative.

In the present study, a single training session was found to be sufficient to produce CSspecific behavioral frequency generalization functions for respiration and heart rate. Similarly, that presentation of unpaired tone and NB stimulation failed to produce the development of CS-specific increases in behavioral responses indicates that rapid specific memory induction in the Paired group is associative. While both respiration and cardiac activity developed CS-specific increased responses, the former appeared somewhat more CS-specific than the latter. That is, in contrast to respiration, heart rate exhibited CS-specific significance within-groups only for the triad of frequencies centered on the CS, rather than for these three individual frequencies (compare Figs. 7C and 8C). The greater specificity for respiration might reflect the procedure of presenting stimuli based on the stability of this behavior rather than on the basis of background heart rate. The differential degrees of specificity exhibited also demonstrate that there is benefit in obtaining behavioral evidence of learning from more than one response system.

Given the unusual fact that the induction of behavioral memory has been accomplished by pairing a tone with stimulation of the motivationally neutral (see below) nucleus basalis, rather than by using a standard reward or punishment, it is important to bear in mind that the current findings actually transcend the standard criteria for associative memory. Memory accomplished by the use of a standard reward or punishment is considered to be associative when it develops only in a paired conditioning group but not in a proper non-associative control group, even without exhibiting specificity to a CS parameter, such as acoustic frequency. Insofar as the prior (McLin et al., 2002a, 2003) and current findings meet both criteria, indeed yield behavioral generalization gradients that themselves cannot be distinguished from those produced by standard training, NB-induced specific induction of memory is authentic. Indeed, the use of NB stimulation to induce associative memory may prove to be more powerful than standard reward or punishment to the extent that it produces highly CS-specific memory without the need for discrimination training (Mackintosh, 1974).

That is not to say that it is "identical" to memory which develops during standard training conditions. The prior and current experiments are parts of a research program that is concerned with the extent to which memory induced by stimulation of the nucleus basalis has the same characteristics and mechanisms as standard memory. There must be differences because NB-induced memory apparently does not involve overt reward or punishment and their concomitant emotional involvement. However, the similarities may prove more illuminating than their differences. Thus, the nucleus basalis may be normally engaged during the formation of standard associative memory, as well as in NB-induced memory. The current findings also indicate that NB-induced memory may in some sense be more efficacious than memory induced by standard experiences because CS-specificity developed in these cases whereas, e.g., 200 trials of training with a single frequency CS, but in the absence of frequency discrimination training, would not be expected to yield such a high degree of stimulus specificity (Mackintosh, 1974).

\subsection{Comparison to previous findings}

While the current findings using a much briefer training regimen are in accord with and extend the previous findings to rapid induction of memory, other differences from the (McLin et al., 2002a, 2002b) study are worthy of consideration. First, the present experiment obtained behavioral responses to test tones before, as well as after, training. In addition to 
showing that there were no between group differences in response to tones before training, this procedure permitted within-group ("before-after") assessment of training effects. Within-group analyses showed that between group differences involved both increased responding to CS-related frequencies in the Paired group, and also decreased responding to "CS"-related frequencies in the Unpaired group (Figs. 7C and 8C).

Second, the present study used nine, rather than seven, test tones. This permitted grouping of frequencies in triads, one of which was centered on the CS frequency. Such additional analysis revealed within-group increases and decreases in heart rate for the Paired and Unpaired groups, respectively (Fig. 8C). (It also showed a between groups difference for evoked potentials, Fig. 9B.)

Third, the present study employed overt delineation and control of animal state, during which stimuli were given only when subjects were in a stable state of calm waking, with regular respiration and an EEG with much low frequency and little high frequency power (Fig. 4). This state control may have contributed to the difference in the behavior of the control groups. In the present case, the control group exhibited "CS"-specific decreased behavioral responses in contrast to (McLin et al., 2002b) in which the control group exhibited a much less prominent "CS"-specific reduction for heart rate and a flat posttraining frequency generalization gradients for respiration. It seems unlikely that the specific decreases found here were due to the much briefer training, although this possibility cannot be ruled out. It is conceivable that the decreased behavioral responses reflect frequencyspecific habituation that is known to develop in a single session of repeated stimulus exposure (e.g., Condon \& Weinberger, 1991; Sharpless \& Jasper, 1956). Habituation might be faster and more specific when a tone is repeatedly (i.e., 200 times) presented while an animal is in a state of quiet waking. Such learning would not be expected to be efficient during paradoxical sleep due to reduced sensory processing and learning, nor during active exploration, when the tone would compete with numerous other stimuli for adequate processing. Less specificity might have occurred in the previous study if extreme stimulation with 3000 trials presented over a two week period promotes frequency generalization. To the best of our knowledge, this issue has not been directly studied. Alternatively, if NBforced EEG activation were in some sense bothersome to the subjects, the tone could have served as a "safety" signal that indicated a subsequent absence of NB stimulation for at least several seconds.

Fourth, the rapid induction of CS-specific increased behavioral responses might reflect the current approach of training and testing while the subjects were in the same calm state, which may have increased the effectiveness of tone-NB pairing by avoiding ceiling effects of ACh release and by circumventing interference with the expression of original learning by testing it in a different state.

This potential explanation for specific decreased responding in the Unpaired group raises the issue of the motivational neutrality of NB stimulation. One possibility is that the NB seems acts as an effective but neutral cortical activation mechanism (Olds \& Peretz, 1960; Wester, 1971) that is "downstream" of structures that evaluate the hedonic value of primary reinforcers and is more involved with receiving "instructions" to release acetylcholine in response to conditioned signals that anticipate reward or punishment (Maho et al., 1995). The NB is not part of any identified reward system (Pennartz, 1995). Recently, Wilson and $\mathrm{Ma}$ (2004) found that neurons in the cholinergic basal forebrain of the macaque monkey do not respond to rewards but developed responses to stimuli that signaled the availability of reward. In recent studies, we found that place preference tests revealed no preference for or avoidance of specific locations in which NB stimulation was delivered (Miasnikov, Poytress, Chen, \& Weinberger, 2004). Thus, although one cannot yet definitively rule out a 
reward or punishment explanation of the induction of memory by NB stimulation, there seems to be little if any evidence to support this possibility.

\subsection{Evoked potentials and behavior}

The need to record the EEG to define behavioral state and to determine the cortical effects of NB stimulation provided an opportunity to record tone evoked potentials pre-and posttraining. As explained previously, such potentials recorded with an epidural screw electrode do not yield precise frequency tuning curves. Previous studies, using intra-cortical electrodes of higher impedance, have shown highly stable (weeks) tuning of auditory cortical evoked potentials (Galván et al., 2001) and associatively induced CS-specific shifts of frequency tuning (Galván \& Weinberger, 2002). Nonetheless, the present evoked potential data are of interest because they provide within-subject information about the effects of NB stimulation on both the induction of behavioral memory and the induction of plasticity in the auditory cortex.

Training did induce CS-specific changes in evoked potentials. Responses to the CS frequency and its immediately adjacent frequencies developed a between groups difference (Fig. 9B), which was characterized by increased amplitude in the Paired group and decreased amplitude in the Unpaired group. However, within-groups, neither was statistically significant (Fig. 9C).

Nonetheless, correlational analyses across all subjects, for the three frequencies centered on the CS frequency, revealed significant positive correlations between evoked potential changes and changes in respiration and heart rate, $\left(r_{\mathrm{xy}}=.49, p<.02\right.$ and $r_{\mathrm{xy}}=.50, p<.01$, respectively) (Fig. 10). Thus, the greater the increase in evoked potentials, the greater the increase in behavioral responses and the greater the decrease in evoked potentials, the greater the decrease in behavioral responses. This finding suggests that the cortical plasticity and the behavioral memory may share some common mechanisms. It does not by itself indicate any causal relationship between CS-specific auditory cortical plasticity and behavioral memory as indexed by changes in respiration and heart rate.

An increase in EP amplitude following pairing might reflect an increase in responses of existing circuitry processing the events, an increase in synchronized response of those elements, recruitment of additional elements, suppression or desynchronization of nonparticipating elements, or any combination of these processes (Pantev, Dinnesen, Ross, Wollbrink, \& Knief, 2005).

\subsection{General considerations of the auditory cortex and behavior in learning and memory}

The absence of any claim regarding a causal relationship between the cortical plasticity and behavior is well-worth emphasizing because studies of learning and memory, such as the present one, that obtain both neurophysiological plasticity and behavioral validation of learning/memory are very often erroneously assumed to be seeking a "stimulus-response" (S-R) circuit (i.e., conditioned stimulus to conditioned response). Such an unjustified assumption then leads to a negative critique of the findings, based in part on the belief that sites of neurophysiological plasticity must be the sole source of the relevant memory, hence their destruction should prevent learning (Ohl \& Scheich, 2004). In contrast, the evidence indicates that, except for some well-defined stimulus-response (S-R) learning/memory circuits which do not include the cerebral neocortex (Christian \& Thompson, 2005), memory of an event appears to be widely distributed, with each component of the network probably involved in a particular aspect of the entire memory substrate (Weinberger, 2004a). The auditory cortex thus appears to store specific information as part of the distributed 
network. Moreover, this information is almost certainly useful beyond the elicitation of behavioral indices of learning that are used at the moment.

We have discussed at length a critical alternative to the S-R (CS-CR) approach, which seeks the neural mechanisms by which two or more stimuli become associated ( $\mathrm{S}-\mathrm{S}$ or CSUS). Given that associations can be indexed by numerous conditioned responses (Lennartz \& Weinberger, 1992; Winters, McCabe, \& Schneiderman, 2002), many laboratories use convenient rapidly developing conditioned responses as validation that learning has occurred, without reference to the issue of the particular efferent circuitry responsible for a specific behavioral index (e.g., respiration, heart rate, galvanic skin response, blood pressure, conditioned suppression, and freezing) (Weinberger, 1992, 1995; Weinberger et al., 1990). In the case of the primary auditory cortex (or any sensory system), an important goal is to determine how learning alters the on-line processing and stored representation of relevant acoustic information, and the principles by which the brain encodes behaviorally important aspects of the acoustic world. For example, a memory code for the degree of behavioral importance ("value") of a sound was identified in the auditory cortex (Rutkowski \& Weinberger, 2005). In short, studies using various tasks, species, and experimental methods converge on the conclusion that the primary auditory cortex is not a primary sensory analyzer but rather it constructs an updating model of the psychologically relevant acoustic world for each individual (Weinberger, 2004b).

The present findings show that NB-induced specific memory does not require thousands of trials and therefore shares a characteristic with much associative memory in that it can develop rapidly. The fewest number of trials that are sufficient for NB-induced memory remain to be determined, but to date the characteristics of such memory are sufficiently similar to those of memory induced by standard training to support the hypothesis that NB engagement is sufficient to induce specific memory during normal learning.

\section{Future directions}

The current study of the nucleus basalis does not imply that it is an exclusive site for memory induction. Other modulators, as well as acetylcholine, have been implicated in plasticity and memory. For example, norepinephrine alters tuning (Manunta \& Edeline, 1997, 1999), serotonin can regulate intensity-dependent response functions (Juckel, Hegerl, Molnar, Csepe, \& Karmos, 1999) and its levels increase in the ACx during initial stages of avoidance learning (Stark \& Scheich, 1997) while dopamine is involved in the increased representation of a tone paired with stimulation of the ventral tegmental (VTA) reward system (Bao, Chan, \& Merzenich, 2001). Thus, other neuromodulatory systems should be investigated for their ability to induce specific behavioral memory.

While past research on the cholinergic system and learning has focused on neural plasticity, the current findings demonstrate the induction of behavioral memory (not merely plasticity) by activation of the cholinergic nucleus basalis. Thus, they promote the approach of testing hypothesized structures and circuitry by direct intervention, i.e., testing the "mimicry" criterion for memory substrates (Martin et al., 2000). Of course, memory induction by NBs requires further characterization, e.g., whether it exhibits consolidation (increased strength over time in the absence of further pairing) and long-term retention. Mechanisms also need to be elucidated. For example, rapid memory induction permits the short term manipulation of its substrates, e.g., by pharmacological means, providing a means to elucidate its mechanisms in greater detail and better understand the role of the nucleus basalis in normal memory. Thus, while focal, low level stimulation of the nucleus basalis is effective, and stimulation of the nucleus basalis releases acetylcholine into the cortex (Kurosawa, Sato, \& 
Sato, 1989), the present study is consistent with, but does not provide direct information that, this memory induction depends upon cholinergic mechanisms.

\section{Acknowledgments}

We thank Mr. Gabriel K. Hui for assistance with the manuscript and Mrs. Julia Martinson for developing the critical components of Spike-2 data acquisition and analysis software. This research was supported by research grants from the National Institutes of Health, DC-02938 and DC-05592 to N.M.W.

\section{References}

Ashe JH, McKenna TM, Weinberger NM. Cholinergic modulation of frequency receptive fields in auditory cortex: II. Frequency-specific effects of anticholinesterases provide evidence for a modulatory action of endogenous. ACh Synapse. 1989; 4(1):44-54.

Bakin JS, Weinberger NM. Classical conditioning induces CS-specific receptive field plasticity in the auditory cortex of the guinea pig. Brain Research. 1990; 536(1-2):271-286. [PubMed: 2085753]

Bakin JS, Weinberger NM. Induction of a physiological memory in the cerebral cortex by stimulation of the nucleus basalis. Proceedings of the National Academy of Sciences of the United States of America. 1996; 93(20):11219-11224. [PubMed: 8855336]

Bao S, Chan VT, Merzenich MM. Cortical remodeling induced by activity of ventral tegmental dopamine neurons. Nature. 2001; 412(6842):79-83. [PubMed: 11452310]

Barth DS, Kithas J, Di S. Anatomic organization of evoked potentials in rat parietotemporal cortex: Somatosensory and auditory responses. Journal of Neurophysiology. 1993; 69(6):1837-1849. [PubMed: 8394409]

Beninger RJ, Wirsching BA, Jhamandas K, Boegman RJ. Animal studies of brain acetylcholine and memory. Archives of Gerontology and Geriatrics Supplement. 1989; 1:71-89. [PubMed: 2569316]

Bjordahl TS, Dimyan MA, Weinberger NM. Induction of long-term receptive field plasticity in the auditory cortex of the waking guinea pig by stimulation of the nucleus basalis. Behavioral Neuroscience. 1998; 112(3):467-479. [PubMed: 9676965]

Christian KM, Thompson RF. Long-term storage of an associative memory trace in the cerebellum. Behavioral Neuroscience. 2005; 119(2):526-537. [PubMed: 15839799]

Condon CD, Weinberger NM. Habituation produces frequency-specific plasticity of receptive fields in the auditory cortex. Behavioral Neuroscience. 1991; 105(3):416-430. [PubMed: 1863363]

Deutsch JA. The cholinergic synapse and the site of memory. Science. 1971; 174(11):788-794. [PubMed: 4330469]

Dimyan MA, Weinberger NM. Basal forebrain stimulation induces discriminative receptive field plasticity in the auditory cortex. Behavioral Neuroscience. 1999; 113(4):691-702. [PubMed: 10495078]

Dykes, RW. Acetylcholine and neuronal plasticity in somatosensory cortex. In: Steriade, M.; Biesold, D., editors. Brain cholinergic systems. New York: Oxford University Press; 1990. p. 294-313.

Edeline JM, Hars B, Maho C, Hennevin E. Transient and prolonged facilitation of tone-evoked responses induced by basal fore-brain stimulations in the rat auditory cortex. Experimental Brain Research. 1994; 97(3):373-386.

Edeline JM, Maho C, Hars B, Hennevin E. Non-awaking basal forebrain stimulation enhances auditory cortex responsiveness during slow-wave sleep. Brain Research. 1994; 636(2):333-337. [PubMed: 8012817]

Edeline JM, Pham P, Weinberger NM. Rapid development of learning-induced receptive field plasticity in the auditory cortex. Behavioral Neuroscience. 1993; 107(4):539-551. [PubMed: 8397859]

Edeline JM, Weinberger NM. Discrimination training induces selective receptive field (RF) plasticity in the auditory cortex during both easy and difficult tasks. Abs. No. 146.13. Society for Neuroscience Abstracts. 1992; 18(1):339.

Flood JF, Landry DW, Jarvik ME. Cholinergic receptor interactions and their effects on long-term memory processing. Brain Research. 1981; 215(1-2):177-185. [PubMed: 7260586] 
Galván VV, Chen J, Weinberger NM. Long-term frequency tuning of local field potentials in the auditory cortex of the waking guinea pig. Journal of the Association for Research in Otolaryngology. 2001; 2(3):199-215. [PubMed: 11669394]

Galván VV, Weinberger NM. Long-term consolidation and retention of learning-induced tuning plasticity in the auditory cortex of the guinea pig. Neurobiology of Learning and Memory. 2002; 77(1):78-108. [PubMed: 11749087]

Hars B, Maho C, Edeline JM, Hennevin E. Basal forebrain stimulation facilitates tone-evoked responses in the auditory cortex of awake rat. Neuroscience. 1993; 56(1):61-74. [PubMed: 8232918]

Hasselmo ME, Bower JM. Acetylcholine and memory. Trends in Neurosciences. 1993; 16(6):218222. [PubMed: 7688162]

Heffner HE, Heffner RS, Contos C, Ott T. Audiogram of the hooded Norway rat. Hearing Research. 1994; 73(2):244-247. [PubMed: 8188553]

Jasper HH, Tessier J. Acetylcholine liberation from cerebral cortex during paradoxical (REM) sleep. Science. 1971; 172(983):601-602. [PubMed: 4324472]

Ji W, Gao E, Suga N. Effects of acetylcholine and atropine on plasticity of central auditory neurons caused by conditioning in bats. Journal of Neurophysiology. 2001; 86(1):211-225. [PubMed: 11431503]

Ji W, Suga N. Development of reorganization of the auditory cortex caused by fear conditioning: Effect of atropine. Journal of Neurophysiology. 2003; 90(3):1904-1909. [PubMed: 12966181]

Jimenez-Capdeville ME, Dykes RW. Daily changes in the release of acetylcholine from rat primary somatosensory cortex. Brain Research. 1993; 625(1):152-158. [PubMed: 8242394]

Johnston MV, McKinney M, Coyle JT. Evidence for a cholinergic projection to neocortex from neurons in basal forebrain. Proceedings of the National Academy of Sciences of the United States of America. 1979; 76(10):5392-5396. [PubMed: 388436]

Juckel G, Hegerl U, Molnar M, Csepe V, Karmos G. Auditory evoked potentials reflect serotonergic neuronal activity-a study in behaving cats administered drugs acting on 5-HT1A autoreceptors in the dorsal raphe nucleus. Neuropsychopharmacology. 1999; 21(6):710-716. [PubMed: 10633476]

Kilgard MP, Merzenich MM. Order-sensitive plasticity in adult primary auditory cortex. Proceedings of the National Academy of Sciences of the United States of America. 2002; 99:3205-3209. [PubMed: 11880653]

Kilgard MP, Merzenich MM. Cortical map reorganization enabled by nucleus basalis activity. Science. 1998a; 279(5357):1714-1718. [PubMed: 9497289]

Kilgard MP, Merzenich MM. Plasticity of temporal information processing in the primary auditory cortex. Nature Neuroscience. 1998b; 1(8):727-731.

Kilgard MP, Pandya PK, Vazquez J, Gehi A, Schreiner CE, Merzenich MM. Sensory input directs spatial and temporal plasticity in primary auditory cortex. Journal of Neurophysiology. 2001; 86(1):326-338. [PubMed: 11431514]

Konorski, J. Integrative activity of the brain: An interdisciplinary approach. Chicago: University of Chicago Press; 1967.

Kurosawa M, Sato A, Sato Y. Stimulation of the nucleus basalis of Meynert increases acetylcholine release in the cerebral cortex in rats. Neuroscience Letters. 1989; 98(1):45-50. [PubMed: 2565563]

Lennartz RC, Weinberger NM. Analysis of response systems in Pavlovian conditioning reveals rapidly versus slowly acquired conditioned responses: Support for two factors, implications for behavior and neurobiology. Psychobiology. 1992; 20(2):93-119.

Ma X, Suga N. Augmentation of plasticity of the central auditory system by the basal forebrain and/or somatosensory cortex. Journal of Neurophysiology. 2003; 89(1):90-103. [PubMed: 12522162]

Mackintosh, NJ. The psychology of animal learning. New York: Academic Press; 1974.

Maho C, Hars B, Edeline JM, Hennevin E. Conditioned changes in the basal forebrain: Relations with learning-induced cortical plasticity. Psychobiology. 1995; 23(1):10-25.

Manunta Y, Edeline JM. Effects of noradrenaline on frequency tuning of rat auditory cortex neurons. European Journal of Neuroscience. 1997; 9(4):833-847. [PubMed: 9153591] 
Manunta Y, Edeline JM. Effects of noradrenaline on frequency tuning of auditory cortex neurons during wakefulness and slow-wave sleep. European Journal of Neuroscience. 1999; 11(6):2134 2150. [PubMed: 10336682]

Martin SJ, Grimwood PD, Morris RGM. Synaptic plasticity and memory: An evaluation of the hypothesis. Annual Review of Neuroscience. 2000; 23:649-711.

McKenna TM, Ashe JH, Weinberger NM. Cholinergic modulation of frequency receptive fields in auditory cortex: I. Frequency-specific effects of muscarinic agonists. Synapse. 1989; 4(1):30-43. [PubMed: 2672402]

McLin DE III, Miasnikov AA, Weinberger NM. Induction of behavioral associative memory by stimulation of the nucleus basalis. Proceedings of the National Academy of Sciences of the United States of America. 2002a; 99(6):4002-4007. [PubMed: 11904444]

McLin DE III, Miasnikov AA, Weinberger NM. The effects of electrical stimulation of the nucleus basalis on the electroencephalogram, heart rate, and respiration. Behavioral Neuroscience. 2002b; 116(5):795-806. [PubMed: 12369801]

McLin DE III, Miasnikov AA, Weinberger NM. CS-specific gamma, theta, and alpha EEG activity detected in stimulus generalization following induction of behavioral memory by stimulation of the nucleus basalis. Neurobiology of Learning and Memory. 2003; 79(2):152-176. [PubMed: 12591224]

Mesulam MM, Mufson EJ, Levey AI, Wainer BH. Cholinergic innervation of cortex by the basal forebrain: Cytochemistry and cortical connections of the septal area, diagonal band nuclei, nucleus basalis (substantia innominata), and hypothalamus in the rhesus monkey. Journal of Comparative Neurology. 1983; 214(2):170-197. [PubMed: 6841683]

Metherate R, Ashe JH. Basal forebrain stimulation modifies auditory cortex responsiveness by an action at muscarinic receptors. Brain Research. 1991; 559(1):163-167. [PubMed: 1782557]

Metherate R, Ashe JH. Nucleus basalis stimulation facilitates thalamocortical synaptic transmission in the rat auditory cortex. Synapse. 1993; 14(2):132-143. [PubMed: 8392756]

Metherate R, Cox CL, Ashe JH. Cellular bases of neocortical activation: Modulation of neural oscillations by the nucleus basalis and endogenous acetylcholine. Journal of Neuroscience. 1992; 12(12):4701-4711. [PubMed: 1361197]

Metherate R, Tremblay N, Dykes RW. Acetylcholine permits long-term enhancement of neuronal responsiveness in cat primary somatosensory cortex. Neuroscience. 1987; 22(1):75-81. [PubMed: 2888049]

Metherate R, Weinberger NM. Cholinergic modulation of responses to single tones produces tonespecific receptive field alterations in cat auditory cortex. Synapse. 1990; 6(2):133-145. [PubMed: 2237776]

Miasnikov AA, McLin D III, Weinberger NM. Muscarinic dependence of nucleus basalis induced conditioned receptive field plasticity. NeuroReport. 2001; 12(7):1537-1542. [PubMed: 11388444]

Miasnikov, AA.; Poytress, BS.; Chen, JC.; Weinberger, NM. 2004 Abstract Viewer. Washington, DC: Society for Neuroscience; 2004. Stimulation of the nucleus basalis that induces behavioral memory in the rat is motivationally neutral. Prog. No. 207.6.

Morris JS, Buchel C, Dolan RJ. Parallel neural responses in amygdala subregions and sensory cortex during implicit fear conditioning. Neuroimage. 2001; 13(6 Pt 1):1044-1052. [PubMed: 11352610]

Morris JS, Friston KJ, Dolan RJ. Experience-dependent modulation of tonotopic neural responses in human auditory cortex. Proceedings of the Royal Society B: Biological Sciences. 1998; 265(1397):649-657.

Oh JD, Edwards RH, Woolf NJ. Choline acetyltransferase mRNA plasticity with Pavlovian conditioning. Experimental Neurology. 1996; 140(1):95-99. [PubMed: 8682184]

Ohl FW, Scheich H. Differential frequency conditioning enhances spectral contrast sensitivity of units in auditory cortex (field $\mathrm{Al}$ ) of the alert Mongolian gerbil. European Journal of Neuroscience. 1996; 8(5):1001-1017. [PubMed: 8743748]

Ohl FW, Scheich H. Learning-induced dynamic receptive field changes in primary auditory cortex of the unanaesthetized Mongolian gerbil. Journal of Comparative Physiology A: Sensory, Neural and Behavioral Physiology. 1997; 181(6):685-696. 
Ohl, FW.; Scheich, H. Fallacies in behavioral interpretation of auditory cortex plasticity. Nature Reviews Neuroscience. 2004. Published on November 20, 2004, at <http://www.nature.com/nrn/ journal/v5/n4/corres/nrn1366_fs.html>

Olds J, Peretz B. A motivational analysis of the reticular activating system. Electroencephalography and Clinical Neurophysiology. 1960; 12:445-454. [PubMed: 14428620]

Pantev, C.; Dinnesen, A.; Ross, B.; Wollbrink, A.; Knief, A. Dynamics of auditory plasticity after cochlear implantation: A longitudinal study. Cerebral cortex. 2005. Published on April 20, 2005, at <http://www.ncbi.nlm.nih.gov/entrez/query.fcgi? cmd=Retrieve $\& \mathrm{db}=$ PubMed\&dopt=Citation\&list_uids=15843632>

Paxinos, G.; Watson, C. The rat brain: In stereotaxic coordinates. 3. San Diego: Academic Press; 1997.

Pennartz CM. The ascending neuromodulatory systems in learning by reinforcement: Comparing computational conjectures with experimental findings. Brain Research Reviews. 1995; 21(3):219245. [PubMed: 8806015]

Rasmusson DD, Clow K, Szerb JC. Frequency-dependent increase in cortical acetylcholine release evoked by stimulation of the nucleus basalis magnocellularis in the rat. Brain Research. 1992; 594(1):150-154. [PubMed: 1467935]

Rutkowski RG, Weinberger NM. Encoding of learned importance of sound by magnitude of representational area in primary auditory cortex. Proceedings of the National Academy of Sciences of the United States of America. 2005; 102(38):13664-13669. [PubMed: 16174754]

Sharpless S, Jasper H. Habituation of the arousal reaction. Brain. 1956; 79(4):655-680. [PubMed: 13396069]

Shaw NA. The auditory evoked potential in the rat-a review. Progress in Neurobiology. 1988; 31(1): 19-45. [PubMed: 3287454]

Shaw NA. A simple technique for localizing the primary auditory cortex in the rat. Physiology and Behavior. 1990; 48(1):207-209. [PubMed: 2236273]

Sokolov, EN. Perception and the conditioned reflex. Oxford, England: Moscow University Press; 1958.

Stark H, Scheich H. Dopaminergic and serotonergic neuro-transmission systems are differentially involved in auditory cortex learning: A long-term microdialysis study of metabolites. Journal of Neurochemistry. 1997; 68(2):691-697. [PubMed: 9003057]

Stratton LO, Petrinovich L. Post-trial injections of an anti-cholinesterase drug and maze learning in two strains of rats. Psychopharmacologia. 1963; 26:47-54. [PubMed: 14082381]

Thiel CM, Friston KJ, Dolan RJ. Cholinergic modulation of experience-dependent plasticity in human auditory cortex. Neuron. 2002; 35(3):567-574. [PubMed: 12165477]

Tremblay N, Warren RA, Dykes RW. Electrophysiological studies of acetylcholine and the role of the basal forebrain in the somatosensory cortex of the cat. II. Cortical neurons excited by somatic stimuli. Journal of Neurophysiology. 1990; 64(4):1212-1222. [PubMed: 2258742]

Webster HH, Rasmusson DD, Dykes RW, Schliebs R, Schober W, Bruckner G, et al. Long-term enhancement of evoked potentials in raccoon somatosensory cortex following co-activation of the nucleus basalis of Meynert complex and cutaneous receptors. Brain Research. 1991; 545(1-2): 292-296. [PubMed: 1860051]

Weinberger, NM. Beyond neuronal excitability: Receptive field analysis reveals that association specifically modifies the representation of auditory information. In: Squire, LR.; Butters, N., editors. Neuropsychology of Memory. 2. New York: Guilford Press; 1992. p. 496-509.

Weinberger NM. Dynamic regulation of receptive fields and maps in the adult sensory cortex. Annual Review of Neuroscience. 1995; 18:129-158.

Weinberger NM. The nucleus basalis and memory codes: Auditory cortical plasticity and the induction of specific, associative behavioral memory. Neurobiology of Learning and Memory. 2003; 80(3): 268-284. [PubMed: 14521869]

Weinberger, NM. Correcting misconceptions of tuning shifts in auditory cortex. Nature Reviews Neuroscience. 2004a. Published on November 20, 2004, at <http://www.nature.com/nrn/journal/ v5/n4/corres/nrn1366_fs.html> 
Weinberger, NM. Experience-dependent response plasticity in the auditory cortex: Issues, characteristics, mechanisms and functions. In: Parks, TN.; Rubel, EW.; Popper, AN., editors. Plasticity of the auditory system. New York: Springer-Verlag; 2004b. p. 173-228.

Weinberger NM. Specific long-term memory traces in primary auditory cortex. Nature Reviews Neuroscience. 2004c; 5(4):279-290.

Weinberger, NM.; Ashe, JH.; Metherate, R.; McKenna, TM.; Diamond, DM.; Bakin, JS., et al. Neural adaptive information processing: A preliminary model of receptive field plasticity in auditory cortex during Pavlovian conditioning. In: Gabriel, MR.; Moore, J., editors. Learning and computational neuroscience: Foundations of adaptive networks. Cambridge, MA: MIT Press; 1990. p. 91-138.

Wester K. Habituation to electrical stimulation of the thalamus in unanaesthetized cats. Electroencephalography and Clinical Neurophysiology. 1971; 30(1):52-61. [PubMed: 4099538]

Wilson DA, Sullivan RM. Respiratory airflow pattern at the rat's snout and an hypothesis regarding its role in olfaction. Physiology and Behavior. 1999; 66(1):41-44. [PubMed: 10222471]

Wilson FA, Ma YY. Reinforcement-related neurons in the primate basal forebrain respond to the learned significance of task events rather than to the hedonic attributes of reward. Cognitive Brain Research. 2004; 19(1):74-81. [PubMed: 14972360]

Winters, RW.; McCabe, PM.; Schneiderman, N. Functional utility and neurobiology of conditioned autonomic responses. In: Moore, JW., editor. A neuroscientist's guide to classical conditioning. New York: Springer-Verlag; 2002. p. 46-85. 


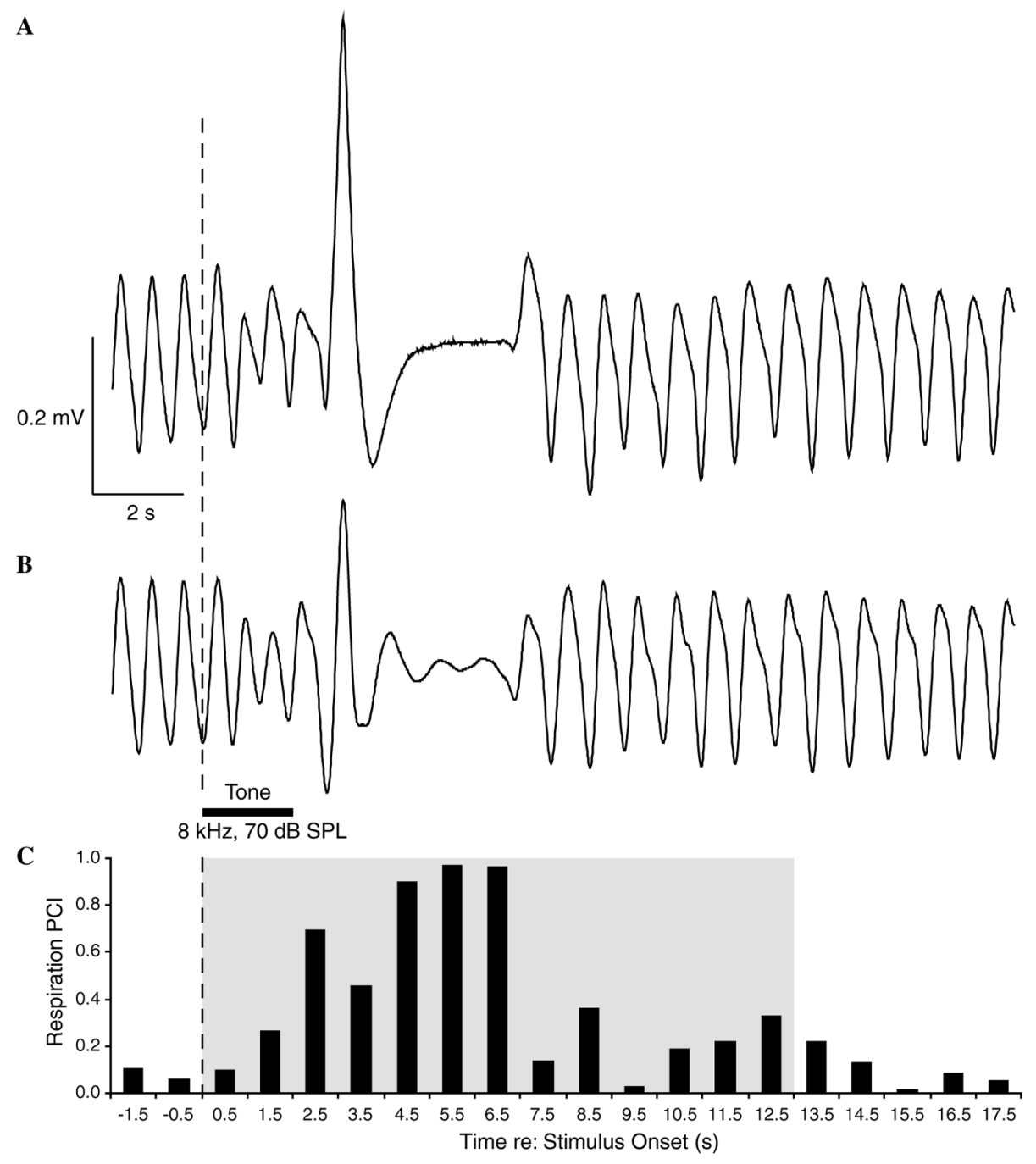

Fig. 1.

Respiration signal and quantification. (A) An example of a regular sinusoidal baseline respiration record disrupted by onset of tone presentation (vertical dotted line in (A) and (B)). In this record, disruption was manifested by a decline in the depth of breathing (1.5$2.5 \mathrm{~s}$ after tone onset), a strong unitary exhalation (at $3.5 \mathrm{~s}$, large positive spike in waveform), and the complete cessation of breathing (4.5-6.5 s latency). Time and amplitude calibrations apply to panels A and B. (B) A portion of the record following FFT analysis $(0.975-2.925 \mathrm{~Hz})$ for further RPCI calculation and processing. (For illustrative purposes, the original waveform (A) was digitally filtered with the same band-pass.) (A) Horizontal black bar denotes tone presentation (2 s) for (A) and (B) panels. (C) Quantification of the respiration record in (B). The "respiration power change index" (RPCI, see Section 2) is sensitive to both increases and decreases in signal amplitude and frequency. The shaded area indicates the $13 \mathrm{~s}$ quantified portion of the respiratory record. 
A

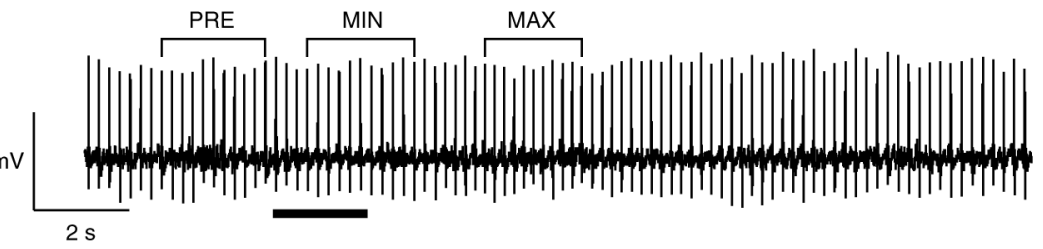

B

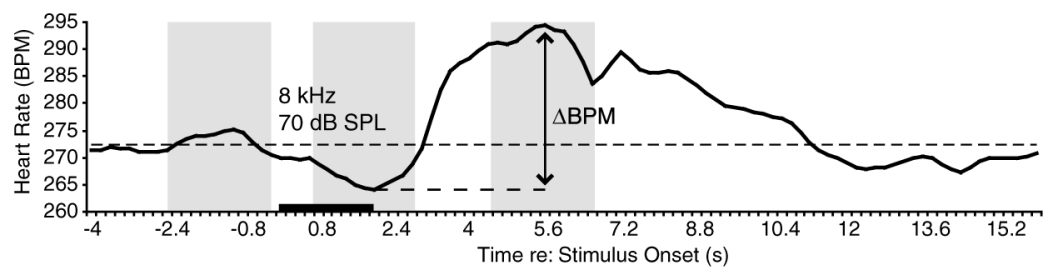

C

PRE

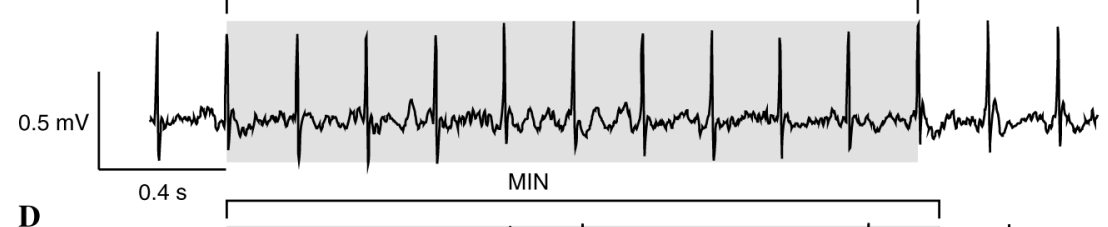

D

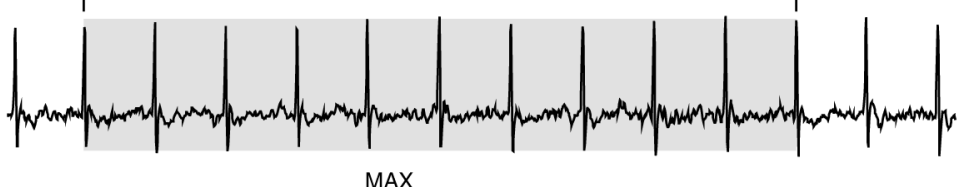

$\mathbf{E}$

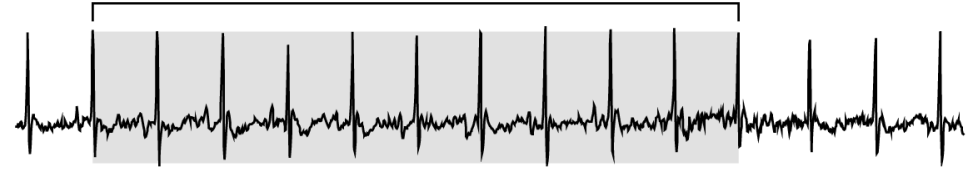

Fig. 2.

Cardiac signal and quantification. (A) Example of a typical biphasic cardiac record (bradycardia followed by tachycardia), delimiting analysis periods: pre-trial (PRE), minimum (MIN), and maximum (MAX) heart rates. Horizontal black bars denote tone presentation in (A) and (B). (B) Quantifica-tion of heart rate; the shaded areas indicate the analysis periods. For each tone presentation, successive inter-beat intervals (IBIs) were calculated and converted into beats per minute (BPM). The difference between the minimum and maximum rates was calculated $(\triangle B P M)$ and analyzed. $(C-E)$ Time expanded plots of the three periods (shaded segments) to permit their visual differentiation. Each segment represents 10 consecutive IBIs for the PRE, MIN, and MAX periods, respectively, aligned with the first heart beat in each segment. Note the longer MIN (D) and the shorter MAX (E) segments of 10 IBIs compared to the PRE period (C). 


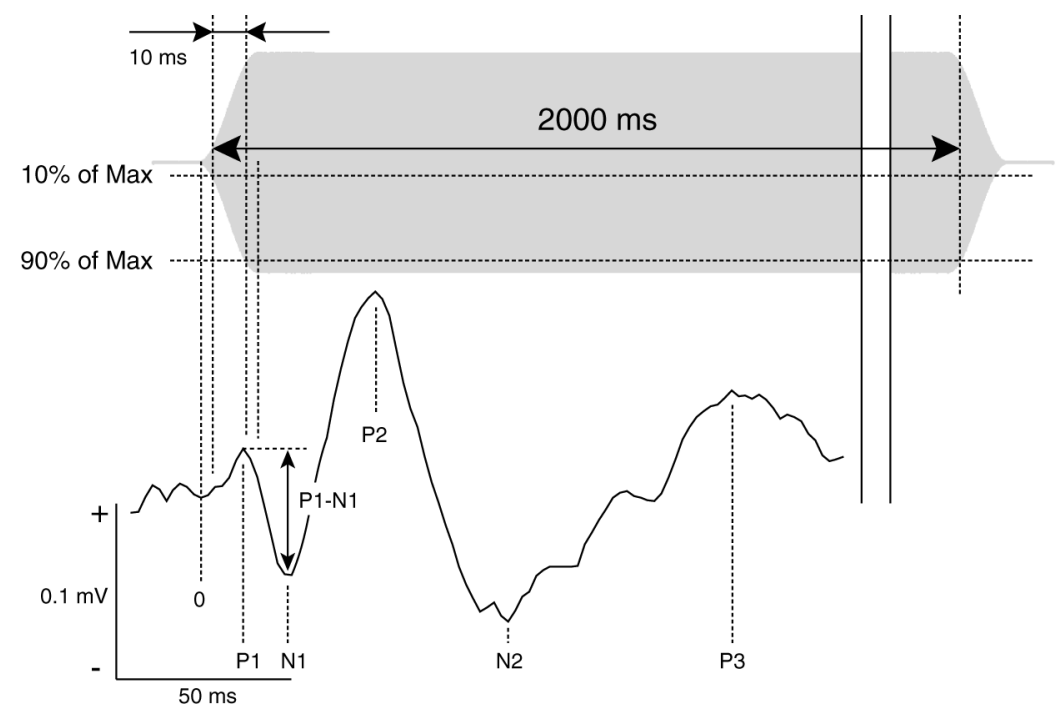

Fig. 3.

Auditory cortical evoked potentials. Top: shaped $(8.0 \mathrm{kHz}, 70 \mathrm{~dB})$ onset and offset portions of $2000 \mathrm{~ms}$ tone stimulus. To avoid startling animals during tone presentations, the rise/fall time was relatively long: a cosine shaped profile with $10 \mathrm{~ms}$ from 10 to $90 \%$ of maximum for rise, and from 90 to $10 \%$ of maximum for fall (actual waveform captured with CED data acquisition system, 50,000 samples/s). Sequential vertical dotted lines (left to right at tone rise) correspond to the tone onset at 0,10,90, and $100 \%$ of maximum, relative to the evoked potential. Bottom: example of an averaged EP (mean of 28 responses to $8.0 \mathrm{kHz}, 70 \mathrm{~dB}$ SPL). The measure of EP amplitude used in analyses was the P1-N1 difference (vertical arrow; see text for details). 
A

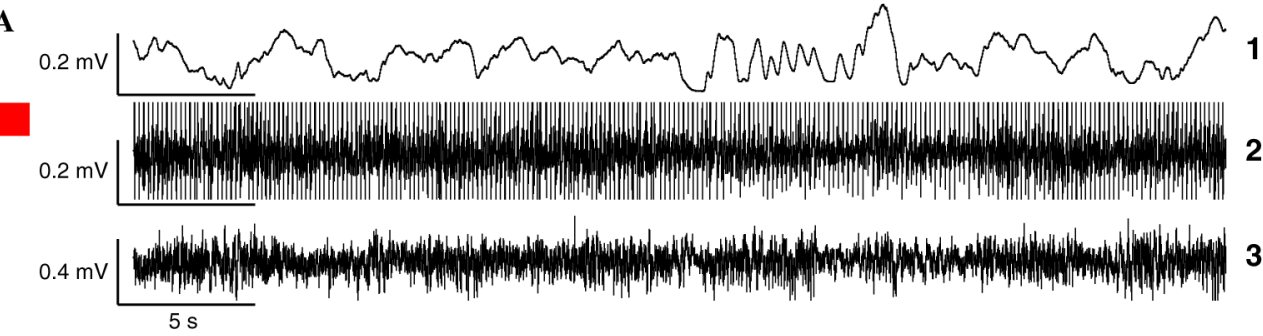

B

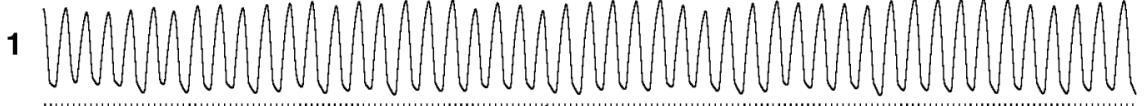

2

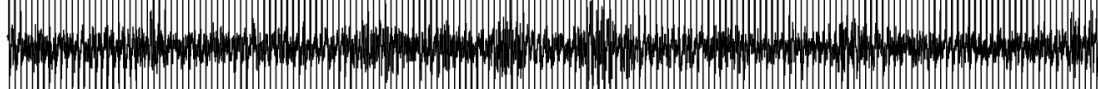

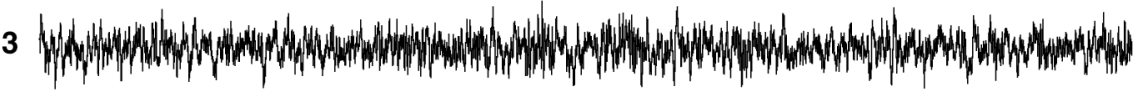

C

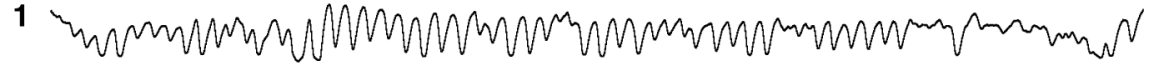

2

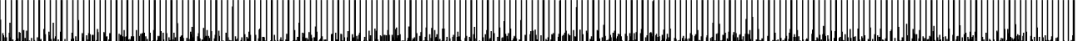

2

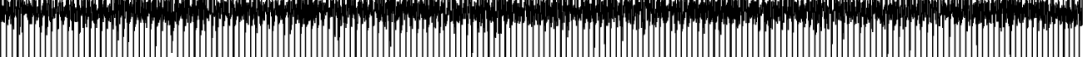

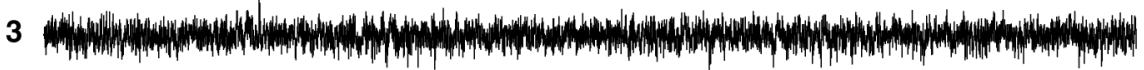
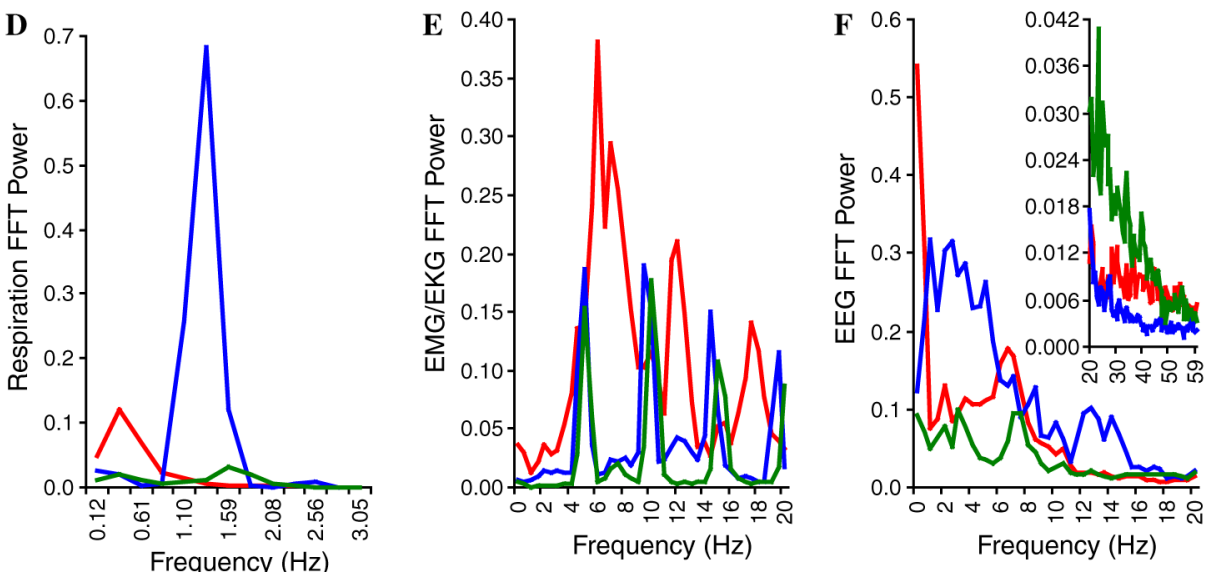

Fig. 4.

Animal states. Examples of different states as defined by respiration pattern (1), EKG/EMG activity (2), and EEG (3). [EKG activity has high amplification (therefore "amplitude clipped") to permit visualization of intermixed EMG activity.] (A) Active exploratory and grooming behavior (video-monitoring) were accompanied by irregular respiration that was mostly high amplitude, low frequency (A1), irregular heart rate and high amplitude EMG activity (A2), and EEG with prominent very slow delta $(<2 \mathrm{~Hz})$ waves and marked theta waves (A3). (B) Calm wakefulness, evidenced by opened-eyed motionless supine posture was accompanied by highly regular high amplitude respiration (B1), with generally lower and more regular EMG/EKG activity (B2), and irregular, although mostly lower frequency (1-8 Hz), often higher amplitude EEG rhythms (B3). (C) Paradoxical (REM) sleep (closedeyes, motionless with occasional twitches) was characterized by low amplitude, shallow mixed regular-irregular respiration $(\mathrm{C} 1)$, lowest amplitude EMG and somewhat regular 
EKG (C2) and lowest amplitude EEG with reduced slow waves (delta, theta, and alpha) and the highest level of high frequency waves (C3). (D-F) Spectral analyses of respiration, EMG/EKG and EEG, respectively, for each of the three states (red = exploration, blue = quiet waking, green = paradoxical sleep). (D) Respiration power was maximal at the lower end of frequency range $(0.37 \mathrm{~Hz})$ during exploration/grooming (red), at both lower $(0.37$ $\mathrm{Hz})$ and higher $(1.59 \mathrm{~Hz}$ ) frequencies during paradoxical sleep (green), and by far largest and most consistent in the middle $(1.35 \mathrm{~Hz})$ of the range during calm wakefulness (blue). Such clear differences make respiration a particularly useful indicator for each state. (E) The peaks of the histograms represent heartbeats carrying maximal power. EMG/EKG power was similar between the calm and paradoxical states. Also, heart rate during exploration was less regular as indicated by broader peaks in the frequency spectrum. (F) The EEG spectrum during exploration had high power at the lowest frequencies with clear theta power $(6-8 \mathrm{~Hz})$ and moderate high frequency power (inset). Calm waking had prominent low frequency (up to $20 \mathrm{~Hz}$ ) power with the lowest level of higher frequencies (inset), while paradoxical sleep had the lowest low frequency power but much the largest high frequencies; exploration had very high power at the lowest EEG frequencies but otherwise an intermediate profile for other frequencies. All stimuli were presented during baseline (pre-training), training and post-training periods when respiration was most regular (B1). 
A

\begin{tabular}{|c|c|c|c|}
\hline \multirow[b]{2}{*}{ Day } & \multicolumn{2}{|c|}{ Groups } & \multirow[b]{2}{*}{ Purpose } \\
\hline & Paired & Unpaired & \\
\hline & 1-15 kHz Tones & 1-15 kHz Tones & Adapt Subjects, Stabilize Responses \\
\hline (2) & 1-15 kHz Tones & 1-15 kHz Tones & $\begin{array}{l}\text { Obtain Baseline Responses } \\
\text { (Pre-Training Behavioral Frequency } \\
\text { Generalization Gradient, BFGG) }\end{array}$ \\
\hline (3) & Tone $\rightarrow$ NBs Paired & Tone, NBs Unpaired & Training \\
\hline (4) & 1-15 kHz Tones & 1-15 kHz Tones & $\begin{array}{l}\text { Evaluate Effects of Initial Training } \\
\text { (Post-Training BFGG) }\end{array}$ \\
\hline$(3$ & Tone, NBs Unpaired & & $\begin{array}{l}\text { Determine Magnitude of Effect } \\
\text { of NBs on EEG }\end{array}$ \\
\hline
\end{tabular}

B Test Tones Only $(1,2,4)$

Paired (3)
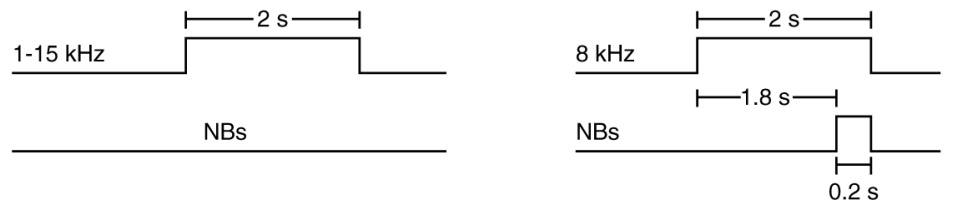

Unpaired $(3,5)$

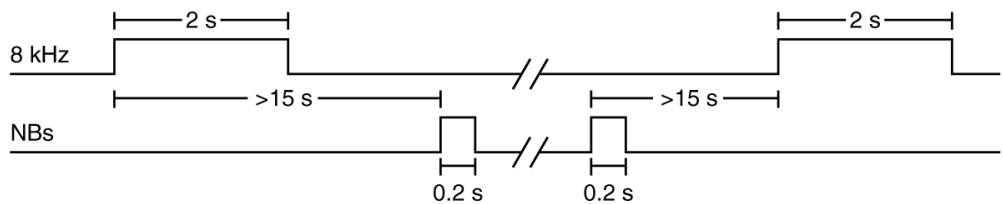

Fig. 5.

Experimental design. (A) The four main stages (days) of the experiment used to obtain pretraining and post-training behavioral generalization gradients for the Paired and Unpaired groups. A fifth stage was used to determine the effectiveness of NB stimulation alone for the Paired group, by shifting it from paired to unpaired tone and NBs. (B) Detailed relationships for presentation of tones alone (Days 1,2, and 4) and stimuli during training (paired and unpaired on Day 3 for the two groups, respectively and Day 5 for the previously Paired group). 
A

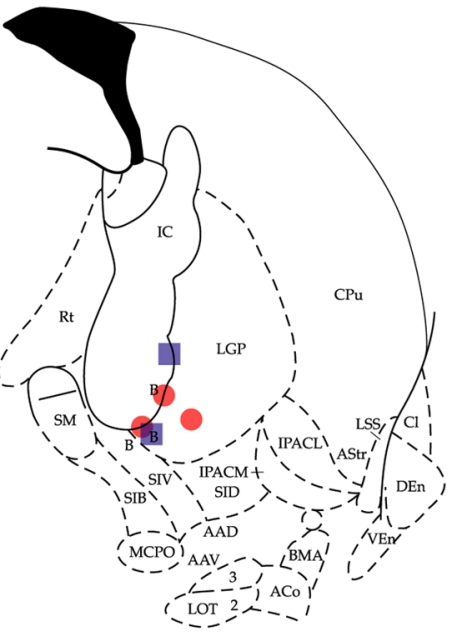

Bregma $-1.30 \mathrm{~mm}$
B

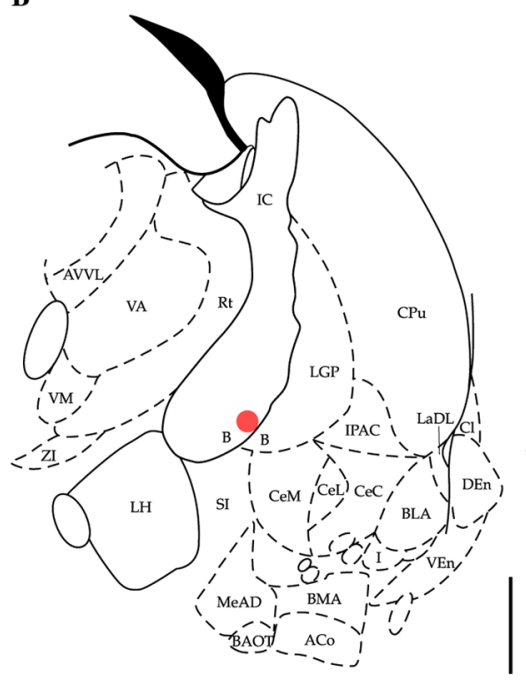

Bregma $-1.80 \mathrm{~mm}$

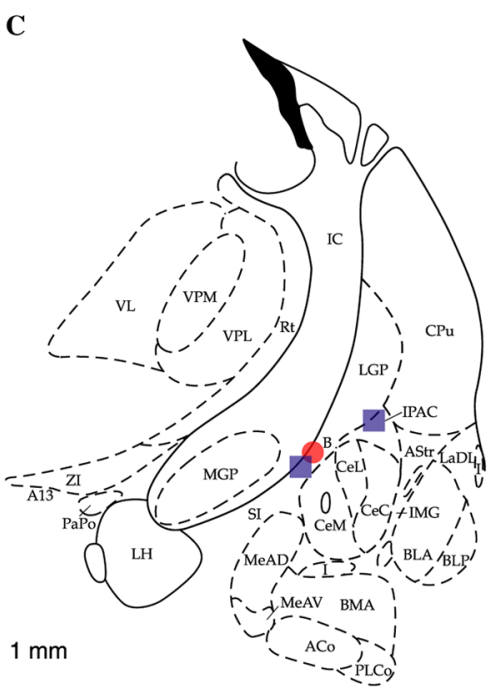

Bregma $-2.30 \mathrm{~mm}$

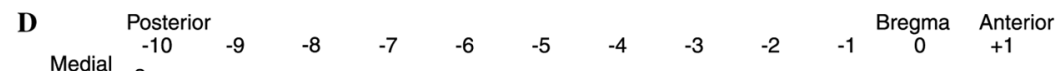

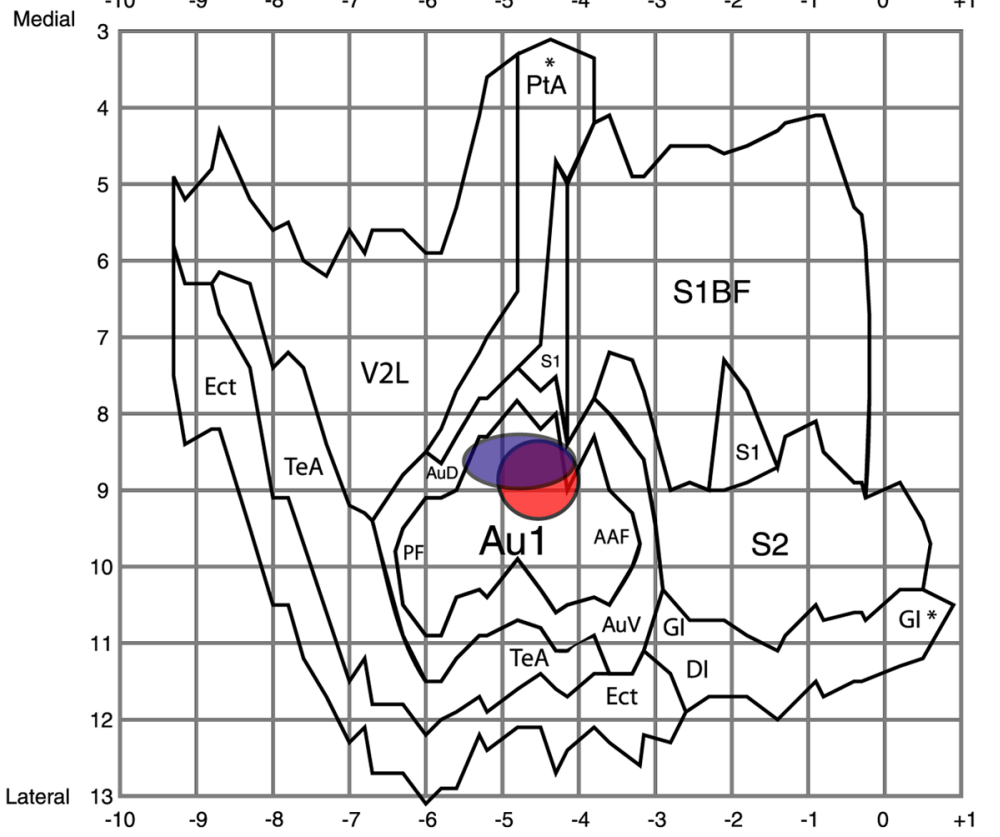

Group P Group Up

Fig. 6.

Stimulating and recording loci. (A-C) Sites of nucleus basalis stimulation projected onto outlines of frontal sections at different anterior to posterior (AP) distances from Bregma (Paxinos \& Watson, 1997). Stimulation was within the caudal nucleus basalis (ventolateral internal capsule, ventromedial lateral globus pallidus and nucleus basalis of Meynart) which projects preferentially to the auditory cortex. There was no significant difference in sites for the Paired (red) and Unpaired (blue) groups ( $t$-tests for all three axes, see Section 3 for details). (D) Recording sites (epidural screw electrodes), projected onto a "flattened" diagram of the temporal cortex (derived from Paxinos and Watson, 1997). The centers of red and blue ellipses correspond to means, and the peripheries of ellipses to standard deviations of locations of recording sites of Paired and Unpaired groups of animals, respectively. There 
were no sig-nificant differences between the groups, either in anterior to posterior ( $X$-axis), or in medio-lateral ( $Y$-axis) directions ( $t$ tests, see Section 3 for details). Note that electrodes were located over the primary auditory cortex. The anterior to posterior and medial to lateral divisions of the grid are shown in mm. Relevant abbreviations for all panels: (see Paxinos and Watson, 1997 for others). (A-C) B, basal nucleus of Meynert; CeM, amygdala central nucleus medial; $\mathrm{CeL}$, amygdala central nucleus lateral; $\mathrm{CPu}$, caudate-putamen; IC, internal capsule; IPAC, interstitial nucleus of posterior limb of anterior commissure; LGP, lateral globus pallidus; LH, lateral hypothalamus; SI, substantia innominata; SIB, substantia innominata, basal; SIV, substantia innomi-nata, ventral; (D) Au1, primary auditory cortex; $\mathrm{AAF}$, anterior auditory field; $\mathrm{AuD}$, secondary auditory cortex, dorsal; AuV, secondary auditory cortex, ventral; PF, posterior auditory field, S1, primary somatosensory cortex; S2, secondary somatosensory cortex; TeA, temporal association cortex; V2L, secondary visual cortex, lateral area. 

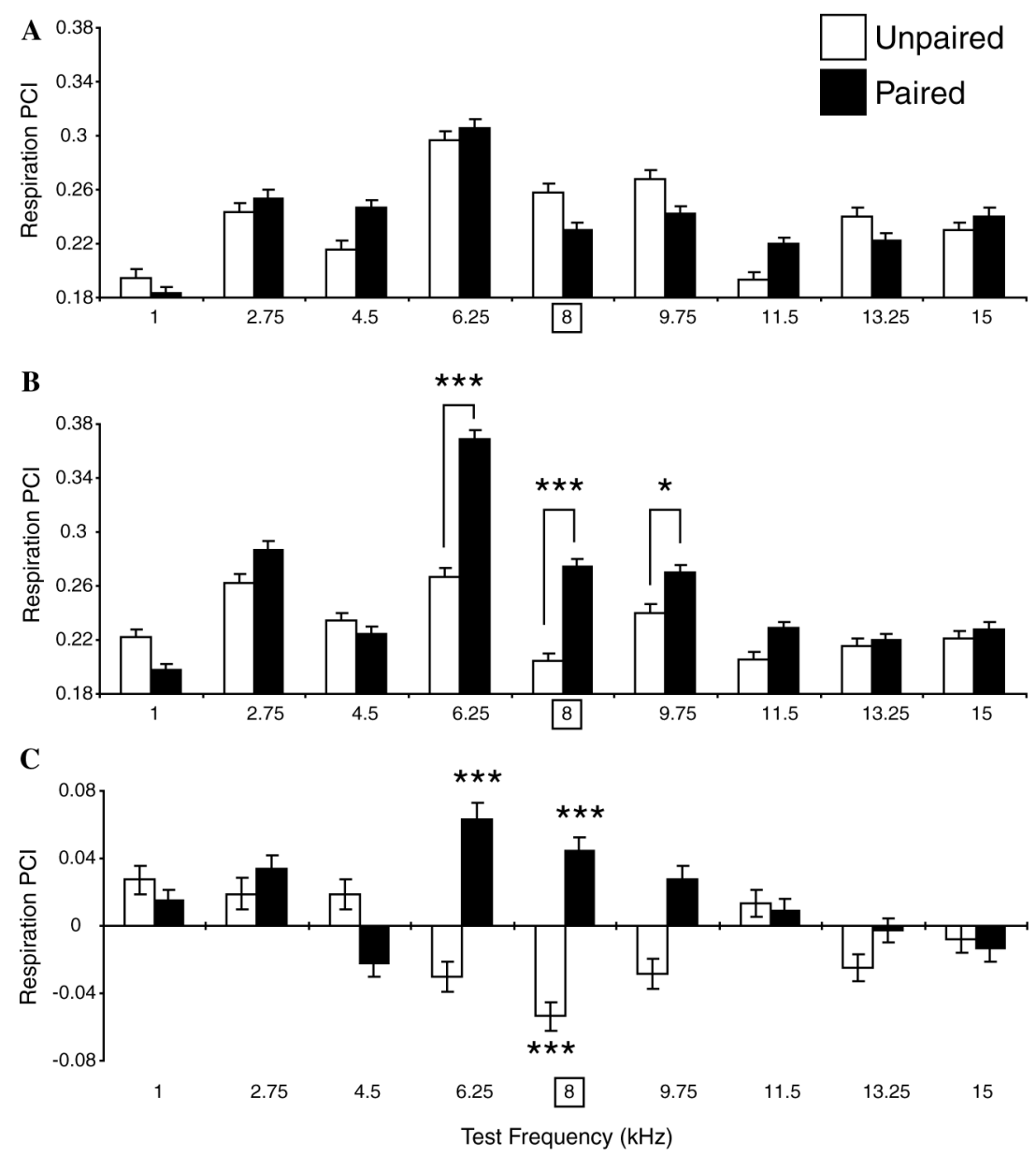

Fig. 7.

Respiration frequency generalization gradients. Average responses (RPCI) to test tones $( \pm \mathrm{SE})$ for all frequencies $(X$-axis, square frame for $8 \mathrm{kHz}$ denotes $\mathrm{CS}$ frequency during training) in both groups (Paired $=$ black bars, Unpaired $=$ white bars) at baseline (pretraining) (A), post-training (B), and within-group differences (C). (A) During the pretraining period, tones in the range of $4.5-9.75 \mathrm{kHz}$ elicited the largest interruptions of respiration, consistent with greater tone sensitivity in this general range as indexed by behavioral audiograms (see text). However, there was no significant difference between groups, providing for an adequate pre-training baseline. (B) Post-training, generalization gradients exhibited CS-specific changes between groups $(p<.001$, ANOVA). The CS (8.0 $\mathrm{kHz})$ and immediately adjacent frequencies $(6.25$ and $9.75 \mathrm{kHz})$ elicited significantly larger responses in the Paired group vs. the Unpaired group. (C) The within-groups comparisons (post minus pre-training) showed that the Paired group developed significant increases of respiratory response at the CS and immediately adjacent frequencies; there was also an unexplained increased response at $2.75 \mathrm{kHz}$. The Unpaired group developed opposite changes, i.e., decreased responses, at and adjacent to the "CS" frequency, which was significant for the "CS" frequency. In this and subsequent figures, statistically significant paired comparisons (post hoc Tukey tests) are indicated by asterisks: $* p<.05, * * p<.01$, $* * * p<.005$, in this and subsequent figures. 

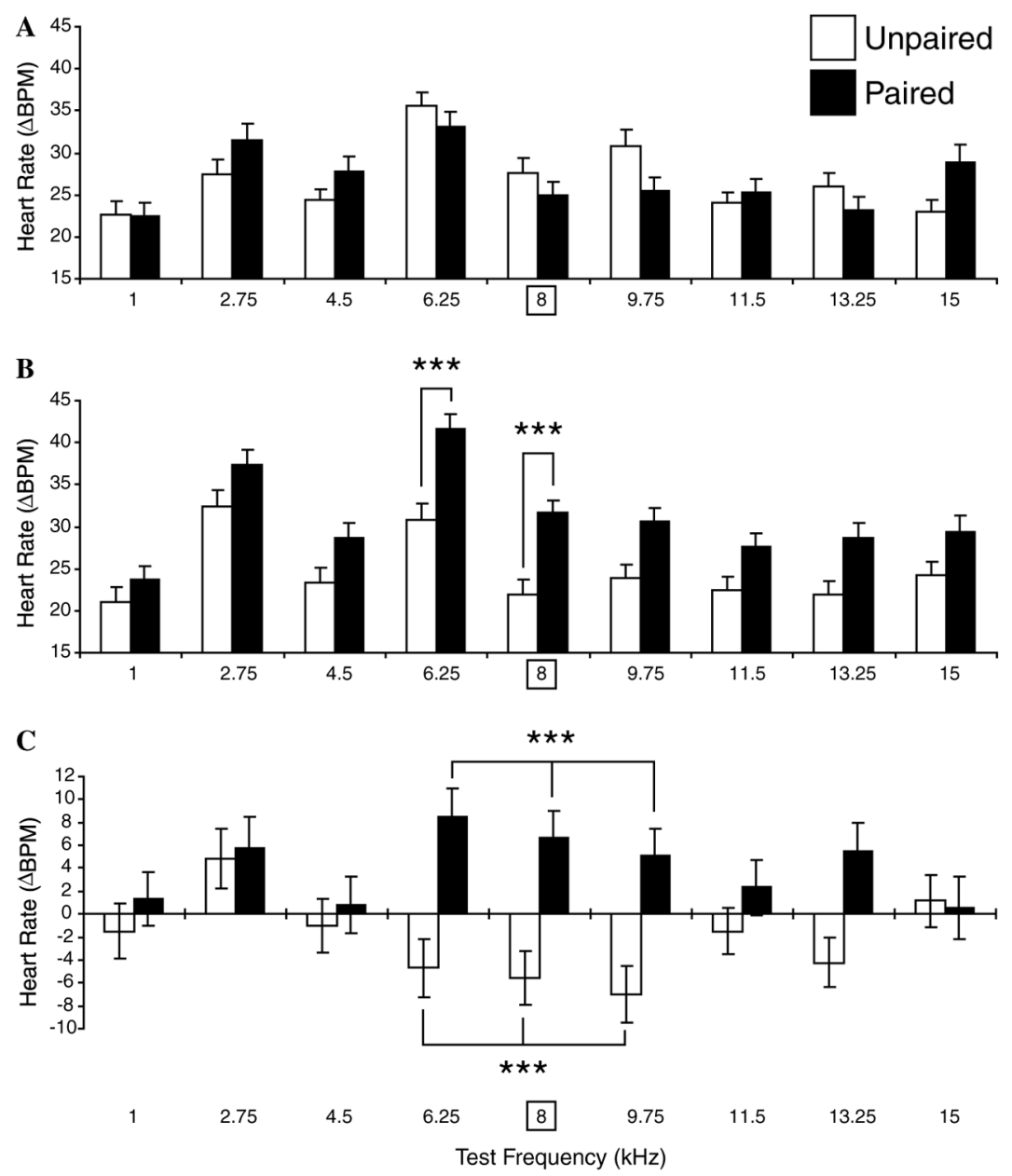

Fig. 8.

Heart rate frequency generalization gradients. Average cardiac responses (changes in beats/ min, $\triangle \mathrm{BPM}$ ) to test tones $( \pm \mathrm{SE})$ for all frequencies and both groups (Paired = black bars, Unpaired = white bars) at baseline (pre-training) (A), post-training (B), and within-group differences $(\mathrm{C})$. (A) The groups did not differ in response to tones during the pre-training baseline period. (B) Cardiac generalization gradients were significantly different after training ( $p<.001$, ANOVA). The differences were confined to the $8.0 \mathrm{kHz}$ CS frequency and its immediately adjacent neighboring frequency of $6.25 \mathrm{kHz}$. (C) Within-groups comparison of the pre- and post-training responses did not reach statistical significance for any single frequency but grouping the nine test frequencies into consecutive subgroups of three showed that pairing produced significant increases in cardiac responses to frequencies centered on the CS while unpaired stimulus presentations produced significant decreases in these same frequencies. The former indicate that pairing induced CS-specific memory while the latter may index frequency-specific habituation. In this and Fig. 9, three-pronged forks denote statistically significant change in the designated triad of frequencies. 

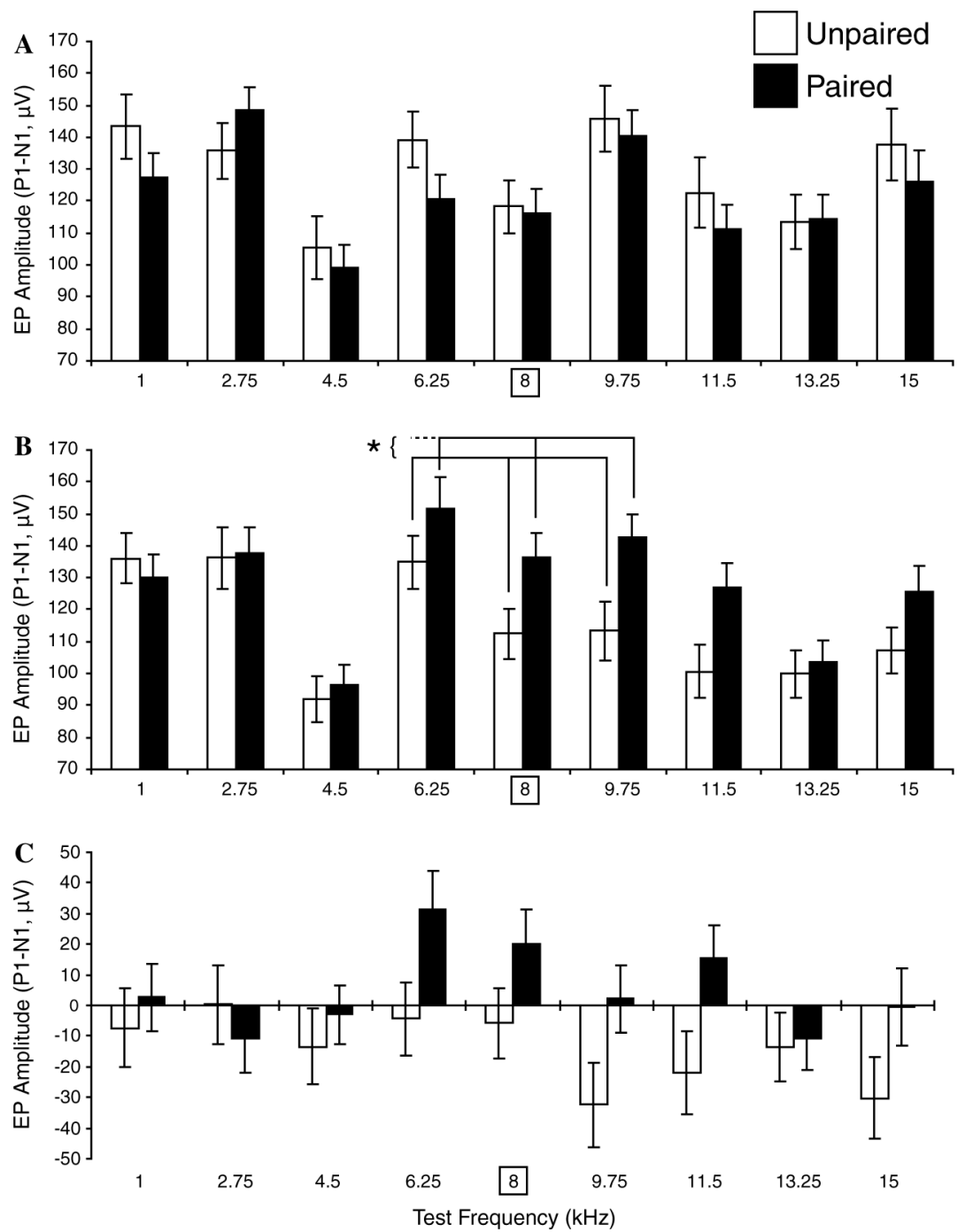

Fig. 9.

Auditory evoked potentials. Average evoked potentials $( \pm \mathrm{SE})$ for all test tones. (Panels and group designations are the same as in Figs. 7 and 8.) (A) Pre-training, while the magnitude of EP responses to tones differed across frequency (see text), there was no significant difference between the Paired and Unpaired groups. (B) During the post-training period, the groups responses were significantly different ( $p=.001$, ANOVA). CS-specific differences were evident. For example, pre-training responses to the CS and its two adjacent frequencies happened to be smaller in the Paired vs. the Unpaired group but became larger than the Unpaired group post-training. However, no single frequency comparisons were statistically significant. Grouping frequencies did reveal a significant difference centered on the CS frequency; responses to the $6.25,8.0$, and $9.75 \mathrm{kHz}$ triad were significantly larger in the Paired group. (C) The within-groups comparison of the pre- and post-training responses exhibited different patterns for the two groups: the Paired group exhibited increased responses around the CS frequency while the Unpaired group exhibited decreased responses at almost all frequencies. However, neither individual nor grouped frequency differences were statistically significant. 

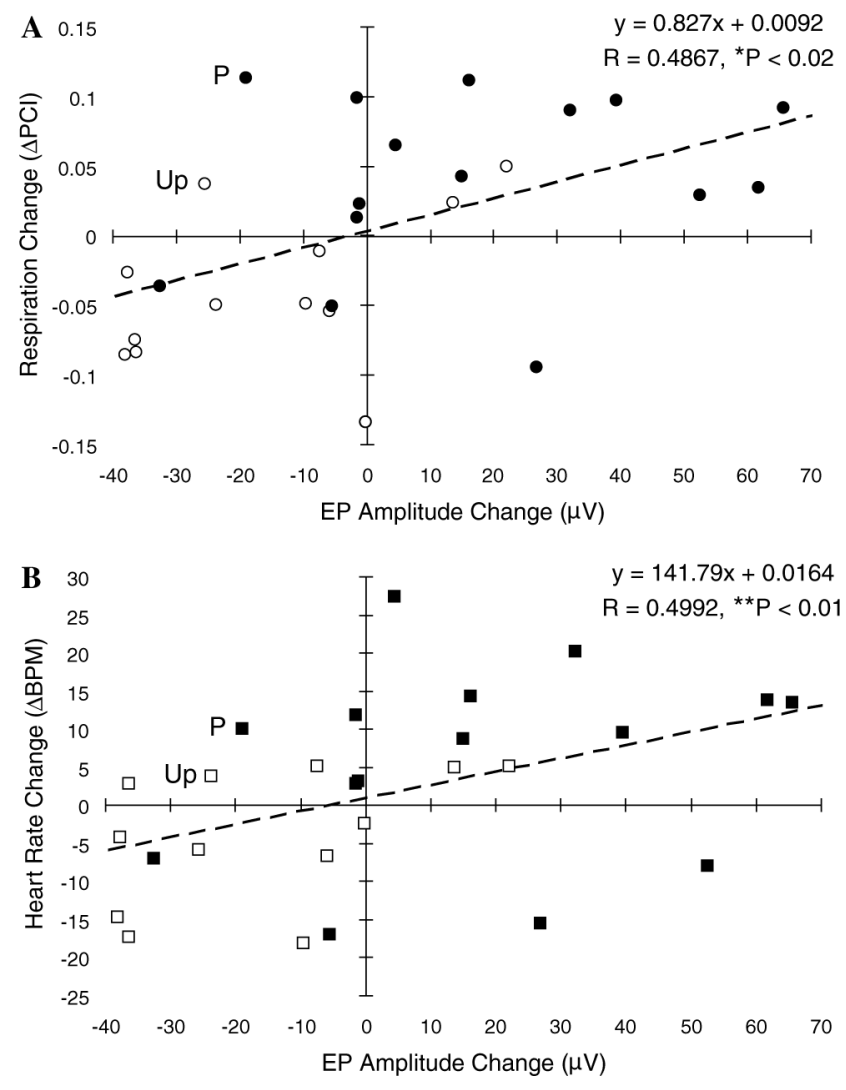

Fig. 10.

Correlation between evoked potentials and behaviors. Scatter-grams showing the relationships between training-induced changes in the amplitude of auditory cortical evoked potentials and changes in respiration responses (A) and heart rate (B), respectively. Paired group $=$ closed circles and squares, Unpaired group $=$ open circles and squares. Both measures show statistically significant positive correlations. 


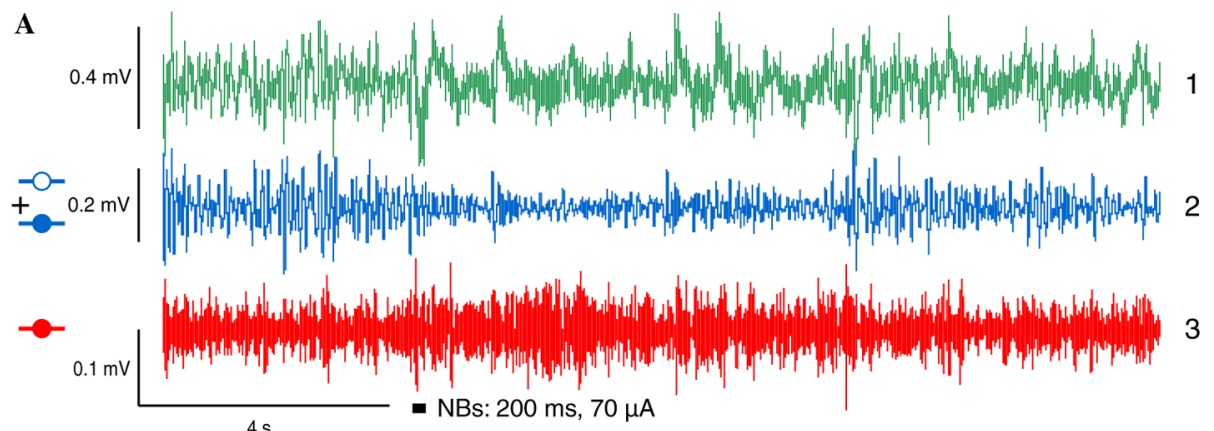

B
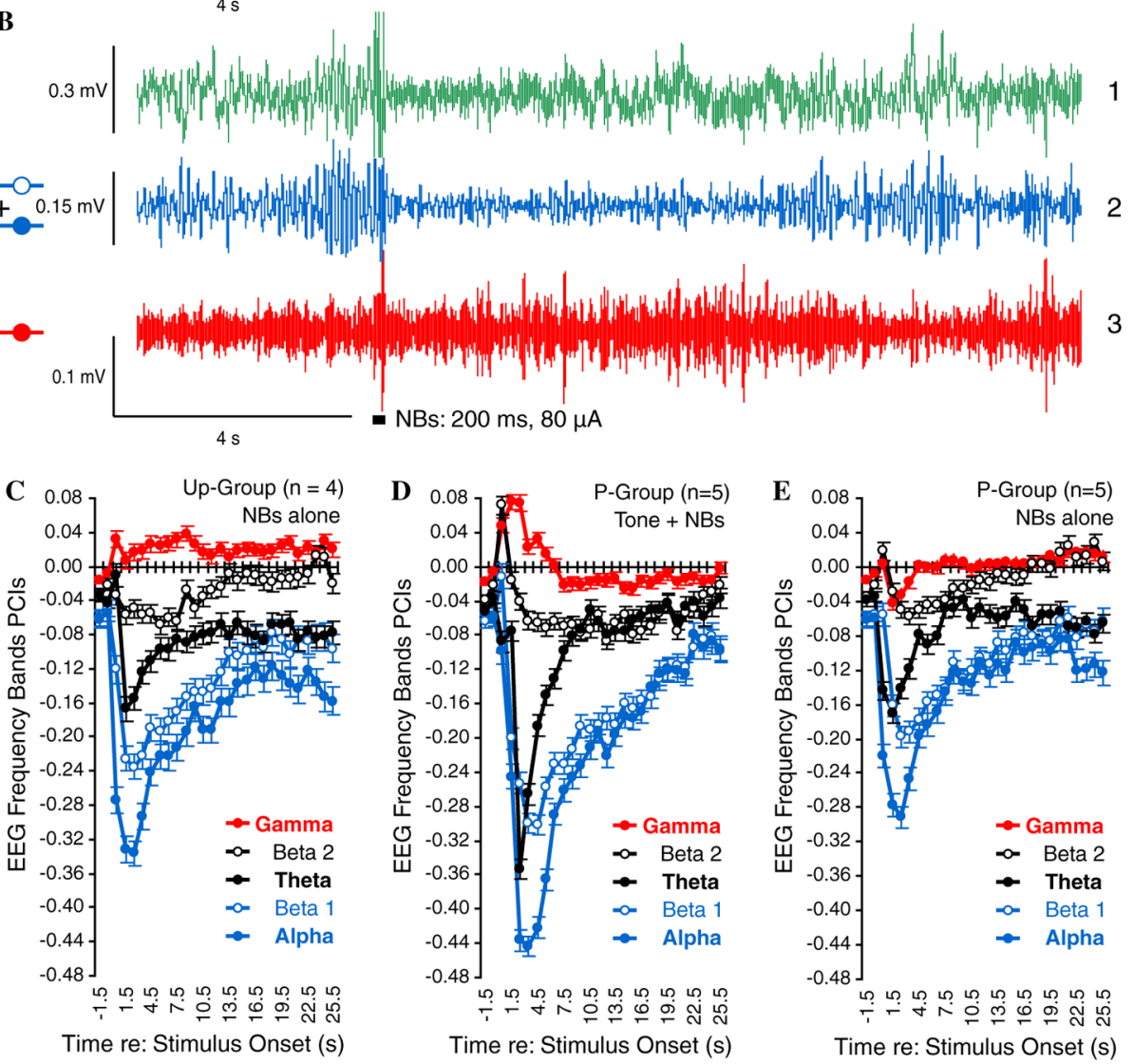

Fig. 11.

Effectiveness of nucleus basalis stimulation. The neurophysiological effectiveness of electrical stimulation of the nucleus basalis was assessed by its modification of the EEG spectrum. (A) and (B) Examples of changes in the EEG following pre-training determination of individual thresholds for a subject in the Unpaired group (A) and the Paired group (B). (A1) and (B1) Original records obtained with band-pass filters set at 1-1000 Hz. (A2) and (B2) Corresponding records band-passed with digital filters set at 8.8-20.0 Hz to emphasize alpha + beta1 bands. (A3) and (B3) Original records filtered at 30-60 Hz to emphasize gamma band: thick horizontal bars indicate NB stimulation. Note the decrease in higher voltage, slower waves [(A1) and (B1)], rendered more clearly by the marked decrease in the power of alpha and beta1 bands (A2) and (B2) and the increase in gamma power (A3) and (B3). (C-E) Changes in EEG spectra in response to NB stimulation. Statistical comparisons were based on the magnitude of maximum change from baseline for each band, computed as the Power Change Index: PCI $=\left(\right.$ Post $_{i}-$ Pre $) /\left(\right.$ Post $_{i}+$ Pre $)$ where the pre-period was the 
mean of the $2 \mathrm{~s}$ immediately preceding NBs and post measures were calculated for consecutive periods of $1 \mathrm{~s}$. (C) Unpaired group: EEG changes during training (NBs presentations). Note increase in gamma band (red) and decrease in beta2 (open black), theta (closed black), beta1 (opened blue), and alpha (closed blue) power. (D) Paired group during presentation of $8.0 \mathrm{kHz}$ tone co-terminating with NBs. This produced even larger changes in all frequency bands, but NBs effectiveness is confounded with preceding conditioned tone evoked EEG effects (McLin et al., 2003). (E) Paired group evaluated during subsequent unpaired presentation of tone and NBs; responses to NB stimulation are shown on the graph (Fig. 5). NB stimulation alone produced EEG changes that were comparable to the NBs effects observed in the Unpaired group, but actually smaller for alpha, beta1, and gamma bands (see text). Thus, the development of CS-specific increased respiratory and cardiac responses in generalization gradients was due to associative processes rather than to stronger effects of NBs on the cortex. 